\title{
Breast cancer risk and serum levels of per- and poly-fluoroalkyl substances: a case-control study nested in the California Teachers Study
}

Susan Hurley ${ }^{1,2^{*}}$ (D) Debbie Goldberg ${ }^{1,2}$, Miaomiao Wang ${ }^{3}$, June-Soo Park ${ }^{3}$, Myrto Petreas ${ }^{3}$, Leslie Bernstein ${ }^{4}$, Hoda Anton-Culver ${ }^{5}$, David O. Nelson ${ }^{1}$ and Peggy Reynolds ${ }^{1,2,6}$

\begin{abstract}
Background: Per- and poly- fluoroalkyl substances (PFASs) are a large family of synthetic chemicals, some of which are mammary toxicants and endocrine disruptors. Their potential as breast carcinogens is unclear. Our objective was to evaluate the risk of breast cancer associated with serum PFAS concentrations in a nested case-control study within the California Teachers Study.

Methods: Participants were 902 women with invasive breast cancer (cases) and 858 with no such diagnosis (controls) who provided $10 \mathrm{~mL}$ of blood and were interviewed during 2011-2015, an average of 35 months after case diagnosis. PFASs were measured using automated online SPE-HPLC-MS/MS methods. Statistical analyses were restricted to six PFASs with detection frequencies $\geq 95 \%$ : PFOA (Perfluorooctanoic acid), PFNA (Perfluorononanoic acid), PFUnDA (Perfluoroundecanoic acid), PFHxS (Perfluorohexane sulfonic acid), PFOS (Perfluorooctane sulfonic acid), and MeFOSAA (2-(N-Methyl-perfluorooctane sulfonamido) acetic acid. Unconditional logistic regression was used to calculate adjusted odds ratios (ORs), estimating the breast cancer risk associated with each PFAS.

Results: For all cases of invasive breast cancer, none of the adjusted ORs were statistically significant but marginally significant ORs $<1.0$ were observed for PFUnDA and PFHxS ( $p$-trend $=0.08$ ). Adjusted ORs $<1.0$ for PFUnDA and PFHXS were statistically significant $(p \leq 0.05)$ among the 107 cases with hormone-negative tumors but not the 743 with hormone-positive tumors.

Conclusion: Overall, these findings do not provide evidence that serum PFAS levels measured after diagnosis are related to breast cancer risk. The few inverse associations found may be due to chance or may be artifacts of study design. Future studies should incorporate information about genetic susceptibility, endogenous estrogen levels, and measurements of PFASs prior to diagnosis and treatment.
\end{abstract}

Keywords: Perfluoroalkyl substances, Polyfluoroalkyl substances, PFAS, Breast cancer risk, Case control

\footnotetext{
* Correspondence: susan.hurley@ucsf.edu

${ }^{1}$ Cancer Prevention Institute of California, Berkeley, CA, USA

${ }^{2}$ Department of Epidemiology and Biostatistics, University of California San

Francisco, 2001 Center Street, Suite 700, Berkeley 94704, California, USA

Full list of author information is available at the end of the article
}

(c) The Author(s). 2018 Open Access This article is distributed under the terms of the Creative Commons Attribution 4.0 International License (http://creativecommons.org/licenses/by/4.0/), which permits unrestricted use, distribution, and reproduction in any medium, provided you give appropriate credit to the original author(s) and the source, provide a link to the Creative Commons license, and indicate if changes were made. The Creative Commons Public Domain Dedication waiver (http://creativecommons.org/publicdomain/zero/1.0/) applies to the data made available in this article, unless otherwise stated. 


\section{Background}

Per- and poly- fluoroalkyl substances (PFASs) are a large family of synthetic chemicals that have been used in the U.S. since the 1950's in a variety of industrial applications and consumer products, including non-stick cookware, food packaging, foam fire retardants, water and stain resistant clothing, carpeting and other textiles [1-3]. Characterized by long, highly fluorinated carbon chains that are extremely resistant to biodegradation, the PFASs are among the most persistent and pervasive environmental pollutants [3-7]. Biomonitoring data indicate human exposures are widespread, with the prevalence of detectable levels of some PFAS approaching $100 \%$ in many populations [3, 8-22]. Although some of the most well-studied and widely-used PFASs were phased out of use or banned in the U.S. and elsewhere earlier this century, these compounds are still used in many areas of the world and there remain at least 3000 other PFASs currently in use worldwide $[2,3,6,23,24]$.

Concern for potential cancer risks stems from a large body of laboratory studies that indicate the potential for myriad toxic effects that could play a role in carcinogenesis, including tumor induction, developmental toxicity, hepatoxicity, immunotoxicity, and endocrine disruption [1, 3, 4, 25-32]. Moreover, a number of reviews recently identified PFASs as priority chemicals of specific concern for breast cancer risk [33-35], based on strong evidence from laboratory studies that indicate that some PFASs may act as estrogen disruptors and mammary gland toxicants [1, 30-32].

Cancer risks in humans, however, remain unclear [36, 37]. Of the thousands of PFASs currently in use, only perfluorooctanoic acid (PFOA) has been evaluated for carcinogenicity by the World Health Organization (WHO) International Agency for Research on Cancer (IARC). Citing "limited" evidence for testicular and renal cancer from human and experimental animal studies that is supported by "moderate" evidence for carcinogenic mechanisms, IARC classified PFOA as a "2B possible human carcinogen" [38, 39]. The United States Environmental Protection Agency (US EPA) in its 2005 draft risk assessment initially classified the evidence for the carcinogenicity of PFOA as "suggestive, but not sufficient," to assess human carcinogenic potential. However, a year later, the US EPA's Science Advisory Board recommended that US EPA consider reclassifying PFOA's carcinogenic potential as "likely to be carcinogenic" [40]. US EPA's final assessment is still forthcoming. Other than PFOA, no other PFASs have been evaluated by health or regulatory agencies to formally classify their carcinogenic potential. A recent review article, however, evaluating the carcinogenic risk of PFOS concluded that the compound could be classified as a group 3 agent "Not classifiable as to its carcinogenicity to humans" in the IARC classification system [36].
Most epidemiologic studies aimed at evaluating cancer risks associated with PFAS exposures have been conducted in occupational cohorts, predominantly comprised of men with insufficient numbers of women to meaningfully examine breast cancer risks [41-48]. Population-based studies are sparse. A pair of analyses from the C8 Science Panel Study conducted in an area of Ohio and West Virginia where the population was known to have long-term high exposures to PFOA due to large-scale environmental releases by a DuPont Teflon manufacturing plant reported statistically significant elevated risks for testicular cancer, as well as suggestively elevated risks for cancers of the kidney, thyroid, and prostate $[49,50]$. Despite large numbers of cases, these studies did not detect any risks for breast cancer associated with increases in estimated cumulative PFOA exposure.

To date, only two sets of studies have been designed specifically to evaluate breast cancer risks associated with PFAS exposures. A case-control study of premenopausal women nested within the Danish National Birth Cohort, while reporting a weakly protective effect for perfluorohexane sulfonic acid (PFHxS) and a weakly elevated risk for perfluorooctane sulfonamide (PFOSA), overall found no consistent statistically significant risks for breast cancer [51, 52]. Analyses from a series of small case-control studies among Greenlandic Inuits, however, reported significantly increased risks for a number of individual and summary measures of PFASs, including PFOSA [51, 53-55]. As suggested by the authors of the Greenlandic study, their positive findings may be due to unusually high PFASs body burdens among Greenlandic Inuits, as well as greater susceptibility to exposures due to polymorphisms in genes involved in estrogen biosynthesis and metabolism common among Greenlandic Inuits [54]. The sparseness of the epidemiologic data in the context of these provocative findings among the Greenlandic Inuits, underscore the need to evaluate the risk of PFASs in other study populations.

The objective of the current study was to evaluate the risk of breast cancer associated with serum levels of several PFASs among participants of a large case-control study, nested within the California Teachers Study (CTS), a statewide prospective cohort established specifically to study breast cancer.

\section{Methods}

\section{Study population}

The study population was comprised of 902 invasive breast cancer cases and 858 controls drawn from the California Teachers Study (CTS). The CTS, an on-going prospective cohort study initiated in 1995-1996 primarily to study breast cancer, is comprised of 133,479 female California public school professionals. Details of the creation and conduct of the CTS are described elsewhere 
[56]. Briefly, since its establishment in 1995-1996 via responses to a mailed questionnaire, the cohort has been followed annually for cancer diagnosis, death, and change of address. State and national mortality files, as well as reports from relatives, are used to ascertain date and cause of death. Address changes for continued follow-up are obtained by several methods including annual mailings, notifications of moves received from participants, and linkages to nationwide consumer reporting companies and the U.S. Postal Service National Change of Address database. Cancer outcomes are identified through annual linkages with the California Cancer Registry (CCR), a legally mandated statewide population-based cancer reporting system. Case ascertainment for the CCR is estimated to be $99 \%$ complete and $99 \%$ of breast cancer tumors are pathologically confirmed [57].

\section{Case and control selection}

Cases and controls included in the present analysis were drawn from CTS members who had provided a blood sample and completed an interview-administered questionnaire as part of their participation in a separately-funded breast cancer case control study nested within the CTS cohort. Case selection criteria for the nested CTS case control study included: diagnosis with invasive breast cancer (SEER Site code $=26,000$ ) between January 1, 2006 and August 1, 2014; age less than 80 years at diagnosis; no prior history of invasive or in situ breast cancer at cohort entry; and a continuous resident of California from cohort entry until time of diagnosis. Controls were drawn from a probability sample of at-risk CTS cohort members frequency matched to breast cancer cases by age at baseline (5-year age groups), race/ethnicity and CCR regional cancer registry of residence. Participation rates were approximately $55 \%$ and $65 \%$ for controls and cases, respectively. Compared to non-participants, participants were slightly older $(89 \%$ versus $86 \%$ aged $\geq 40$ years) and more likely to be white (91\% versus $86 \%$ ). Active refusals were the most common reason for non-participation, with $29 \%$ of controls and $21 \%$ of cases refusing, followed by inability to contact/lack of response (12\% for controls and $9 \%$ for cases). Approximately $4 \%$ of controls and $5 \%$ of cases were excluded due to illness or death. Interview data and blood specimens were collected from 913 invasive breast cancer cases and 1270 controls during the course of the nested CTS study (May 2011 to October 2015).

Prior analyses of serum PFAS levels among controls in the nested CTS study indicated that PFAS levels were highest during the early months of serum collection [58] - a time, by happenstance, when blood samples were disproportionately collected among controls compared to cases. Therefore, to minimize the potential for bias due to the declining temporal trends in PFAS levels, we excluded participants who provided a blood sample prior to October 2011 (this cut point was chosen based upon a sensitivity analysis we conducted to determine the time interval during which sample collection date no longer affected the estimated risk ratios for breast cancer (data not shown)). Furthermore, samples collected during the last 2 months of the CTS nested case control study were not included in the current analysis due to budgetary and timeline constraints of this separately-funded study. Ultimately the participants in the current analyses consisted of all 902 invasive breast cancer cases and 858 controls in the CTS nested case control study who provided a blood specimen and completed an interview-administered questionnaire at blood draw between October 2011 and August 2015. Blood specimens were collected an average of 35 months after case diagnosis (range of interval between diagnosis date and date of specimen collection $=9$ months to 8.5 years).

\section{Serum collection}

Blood was collected, usually in participants' homes, by licensed phlebotomists into a $10 \mathrm{~mL} \mathrm{BD}^{\circ}$ tube (cata$\log \# 367985$, Becton Dickinson, Franklin Lakes, NJ) with clot activator, double gel for transport, and silicone coated interior, using standard phlebotomy techniques. Prior to field processing, specimens were kept on cool packs for at least 30 minutes. Within hours of collection, phlebotomists spun down the clotted blood samples in the field using portable centrifuges to separate the serum portion. Processed samples were then frozen and stored at $-20^{\circ} \mathrm{C}$ for $4-6$ weeks until transported either via local courier (on cool-packs) or overnight (on dry-ice via FedEx) to the laboratory for chemical analysis. Samples remained frozen during this transportation process. Upon receipt at the laboratory, specimens were stored at $-20^{\circ} \mathrm{C}$ until analysis.

\section{Serum PFASs measurements}

Measurement of PFASs in collected sera was conducted using the method as detailed previously [59]. Briefly, $100 \mu \mathrm{L}$ of serum was diluted in formic acid and spiked with isotopically labeled internal standards before injection into the automated on-line solid phase extraction method coupled to liquid chromatography and tandem mass spectrometry (Symbiosis ${ }^{\mathrm{Tm}}$ Pharma, IChrom Solutions, Plainsboro, NJ, and Sciex 4000 QTrap mass spectrometer, Sciex, Redwood City, CA) for clean-up and analysis. Native and isotopically-labeled PFAS standards were purchased from Wellington Laboratories (Shawnee Mission, KS). Within each batch analysis of 20 actual samples, two in-house spiked calf serum samples and NIST 1958 Standard Reference Material were run in duplicate for quality control. The laboratory is proficient in serum PFAS analysis as demonstrated by successful regular participation in proficiency testing (CDC, AMAP). 


\section{Covariate information}

Information on potential covariates was derived from a series of surveys, including a self-administered baseline questionnaire completed in 1995-1996 at the initiation of the CTS, five mailed follow-up surveys, as well as a survey administered by a phlebotomist interviewer at the time of blood draw. Data from the interview administered at blood collection were used to characterize age at blood draw in years (40-49/50-59/60-69/70-79/ $\geq 80$ ), menopausal status (Post/Pre and Peri/unknown), age (in years) at menopause (Pre and Peri-menopausal/ 20-43/44-49/50-51/52-53/54-55/56-65/unknown), years since the onset of menopause (Pre-Peri/unknown-menopausal/0-9/10-19/20-29/30-69/unknown) and hormone therapy (HT) use (never used and post-menopausal/ever used and post-menopausal/Pre and peri-menospausal/unknown). Season of blood collection (Winter $=$ December February; Spring = March - May; Summer $=$ June - Aug; Fall = September-November) was based on the date that the blood sample was collected, as recorded by the phlebotomist. Data from responses to the 1995-1996 baseline questionnaire were used to characterize: age at CTS enrollment in years (20-80); race/ethnicity (White/Black/Hispanic/ Asian/PI/other); body mass index (BMI) (16.0-24.9/25.029.9/30.0-53.3/unknown); physical activity in hours/week (<0.5/0.5-3.9/ $\geq 4.0 /$ unknown); alcohol consumption in grams/day (none/ $<20 / \geq 20 /$ unknown); pack years of smoking (never smoker/ $\leq 10 / 11-20 / 21-30 / \geq 31 /$ unknown); family history of breast cancer (in a first degree relative -- no/yes/ unknown); age at menarche in years $(\leq 11 / 12-13 / \geq 14 /$ never/ unknown); age (in years) at first full-term pregnancy (no full term pregnancy/ $\leq 24 / 25-29 / \geq 30 /$ unknown); breast feeding history (total months) (never pregnant/pregnant without live birth $/ 0 /<6 / 6-11 / \geq 12 /$ unknown). Detailed information on HT use (never/past/current estrogen use/current estrogen and progesterone use/progesterone only use/premenopausal/unknown) was also gathered from the baseline questionnaire. A variable for weight change (loss of $>5$ pounds/ gain of $>5$ pounds/no change of $>5$ pounds/unknown) was calculated based on difference between self-reported weight on the fifth mailed questionnaire (administered in 20122013) and the 1995-1996 baseline questionnaire. Dietary consumption (grams/day) of fat (tertiles/unknown), pork (none/< median/ $\geq$ median/unknown), and total red meat (tertiles/unknown) were derived from information on dietary factors obtained from responses to a modified version of the Block questionnaire included on the 1995-1996 baseline questionnaire [60, 61].

In addition to these survey-based factors, neighborhood characteristics of socioeconomic status and urbanization were also considered as potential covariates as some of our prior work have suggested they may be related to serum PFAS levels [58]. These were derived by linking residence at blood draw to U.S. census data by the block group of residence. A description of these methods appears elsewhere $[62,63]$.

This initial set of potential covariates was chosen to include established breast cancer risk factors and factors that our prior analyses had identified as correlates to serum PFAS concentrations in this study population.

\section{Statistical analysis}

All analyses were conducted in SAS Version 9.4. The limit of detection (LOD) was defined as three-times the standard deviation of the blank. Because the LOD varied by batch, for the purpose of summarization, we calculated the average LOD across all batches, weighted by the number of samples in each batch. Samples with PFAS concentrations below the LOD were imputed as LOD/ $\sqrt{2}[64,65]$. To avoid potential bias from such imputation, six PFASs with detection frequencies (DF) below 95\% were excluded from the risk analyses including: PFOSA (Perfluorooctane sulfonamide), PFBS (Perfluorobutane sulfonic acid), EtFOSSA (2-(N-Ethyl-perfluorooctane sulfonamido) acetic acid), PFDA (Perfluorodecanoic acid), PFDoDA (Perfluorododeconic acid), and PFHpA (Perfluoroheptanoic acid) so that the risk analyses were restricted to the following six PFAS with DFs $\geq$ 95\%: PFOA (Perfluorooctanoic acid), PFNA (Perfluorononanoic acid), PFUnDA (Perfluoroundecanoic acid), PFHxS (Perfluorohexane sulfonic acid), PFOS (Perfluorooctane sulfonic acid), MeFOSAA (2-(N-Methyl-perfluorooctane sulfonamido) acetic acid (Table 2).

Prior to conducting the risk analyses, a number of exploratory and descriptive analyses were conducted. Frequency distributions of potential covariates were evaluated and chi-square statistics were calculated to assess the statistical significance of differences in distribution by case status. Spearman rank correlations between PFASs were calculated. As many of the PFAS were highly correlated we considered each PFAS separately in our analyses. Because the PFAS concentrations were highly-skewed, values were $\log _{10}$-transformed in all subsequent analyses to enable the application of parametric statistical approaches. Smoothing splines were considered in generalized additive models (using PROC GAM) and evaluated to assess potential non-linearities in the relationship between each PFAS and the log-odds of breast cancer but no evidence of non-linearity was observed.

The risk of breast cancer associated with each PFAS was estimated by unconditional logistic regression, using PROC LOGISTIC to generate odds ratios (OR) and 95\% confidence intervals $(95 \% \mathrm{CI})$. PFAS concentrations were considered alternatively in separate sets of models as continuous variables (expressed as $\log _{10}$ [PFAS, ng/ML]) and categorical variables (high/medium/low, based on tertiles of the PFAS concentrations in the controls). All regression models included terms for the matching design variables (age at baseline enrollment, race/ethnicity, 
and region of residence). Crude ORs were calculated from simple models including only these matching design variables. Adjusted ORs were generated from multivariate models, adjusting for potential confounding factors. Starting with the full set of potential covariates (as described in the previous section), covariates for inclusion in the final multivariable models were selected via a two-step process. First, a backwards elimination approach was used, starting with a model that forced inclusion of the PFAS variable, age, race, and region of residence, and retaining all covariates for which the $p$-value for the Wald chi-square was $<0.05$. We then further evaluated potential confounders by adding each of the excluded variables back into the model one at a time and evaluated the effect of the change in the estimated odds ratios for the PFAS. Factors that changed the estimated odds ratio for the PFAS by $10 \%$ or more were retained in our final multivariable regression models. While we conducted this process separately for each PFAS, it resulted in the same set of covariates for all PFASs. The final set of covariates appear as footnotes to Table 3.

A number of stratified analyses were conducted to evaluate whether risks may differ within certain subsets of our study population. The subsets of interest were chosen a priori after an extensive literature review. Selected subsets included types of breast cancer that are thought to have distinct etiologies, including: pre/peri-menopausal versus post-menopausal cases and cases with tumors that were hormonally responsive, identified as estrogen or progesterone receptor positive $(\mathrm{ER}+/ \mathrm{PR}+)$ tumors versus non-hormonally responsive tumors that were estrogen receptor negative and progesterone receptor negative (ER-/PR-). Furthermore, because toxicological evidence suggests that the effects of exposure to xenobiotic endocrine disruptors such as PFASs may differ depending on the endogenous hormonal milieu in which they occur $[66,67]$, we evaluated risks separately for: women who had and had not ever used menopausal HT; nulliparous and parous women; and for women with low, medium and high BMI. Multivariable logistic models were built separately for each of these subset analyses, using the same method described above for covariate selection. Final covariates are listed in the footnotes of Tables 4 and 5 .

\section{Results}

Selected characteristics of the study population are detailed in Table 1. Participants were predominantly middle-aged and older non-Hispanic white women reflecting the characteristics of the CTS cohort from which they were selected. Compared to controls, cases were more likely to report a family history of breast cancer, to smoke cigarettes, have a higher BMI, never had a full-term pregnancy and had a later age at first full-term pregnancy (among parous women), and consume more red meat and pork. Among women who had reached menopause by the time of blood draw, the age at menopause did not differ between cases and controls. Post-menopausal breast cancer cases, however, were slightly more likely than controls to have entered menopause more recently (i.e., within 10 years prior to blood draw). The year and season during which blood draw occurred differed between cases and controls with controls more likely than cases to have had their blood samples collected during the initial few months of the study and during the fall and winter months.

Serum PFAS levels are summarized in Table 2. Summary statistics are not shown for the six PFASs with DFs less than 95\%. Similar to reports from other study populations, concentrations were highest for PFOS, followed by PFOA, and PFHxS. Distributions were generally similar for cases and controls, with Wilcoxon rank sum tests indicating no difference in medians $(p>0.05)$ for all PFASs, with the exception of MeFOSAA for which the median was marginally higher in controls compared to cases $(p=0.04)$.

Statistically significant positive correlations were observed between all the PFASs with Spearman Rank Correlations ranging from 0.21 (for PFHxS and PFUnDA) to 0.63 (for PFOS and PFOA). Correlations between the PFASs were generally similar among cases and controls (data not shown).

The risks associated with serum PFAS concentrations for all invasive breast cancers in our full study population are summarized in Table 3. Overall, the crude ORs, while having marginally more narrow confidence intervals, were similar to the adjusted ORs. All adjusted ORs were close to 1.0 and not statistically significant - regardless of whether the PFAS was modeled continuously or categorically. Marginally significant ORs $<1.0$ for PFHxS and PFUnDA were observed only when modeled as categorical variables ( $p$-value for trend $=0.08$ ) but not when modeled as log-linear continuous variables ( $p=0.18$ and $p=0.25$, respectively).

When stratified by menopausal status, the overall pattern of risk estimates were similar to that observed in the full study population (Table 4). The suggestive inverse associations observed in the full study population for PFHxS and PFUnDA persisted across strata of menopausal status and reached statistical significance for PFUnDA among pre/peri-menopausal women $(\mathrm{p}$-trend $=0.04$ ) and for $\mathrm{PFHxS}$ among post-menopausal women (p-trend $=0.05$ ).

When the data were stratified by tumor hormone responsiveness, no statistically-significant risks were observed for the 743 cases with ER+ or PR+ tumors (Table 5). However, among the 107 cases with tumors that were both ER- and PR-, adjusted ORs significantly 
Table 1 Characteristics of study participants $(n=1760)$, by case-control status

\begin{tabular}{|c|c|c|c|c|}
\hline \multirow[t]{2}{*}{ Characteristic $^{a}$} & \multirow{2}{*}{$\begin{array}{l}\text { Case } \\
\mathrm{N}(\%)\end{array}$} & \multirow{2}{*}{$\begin{array}{l}\text { Control } \\
N(\%)\end{array}$} & \multirow{2}{*}{$\begin{array}{l}\text { All } \\
\mathrm{N}(\%)\end{array}$} & \multirow[t]{2}{*}{$p$-value } \\
\hline & & & & \\
\hline All & $902(100)$ & $858(100)$ & $1760(100)$ & \\
\hline Age at baseline enrollment (years) & & & & 0.51 \\
\hline $20-39$ & $114(13)$ & $89(10)$ & $203(12)$ & \\
\hline $40-44$ & $121(13)$ & $125(15)$ & $246(14)$ & \\
\hline $45-49$ & $204(23)$ & $186(22)$ & $390(22)$ & \\
\hline $50-54$ & $196(22)$ & $198(23)$ & $394(22)$ & \\
\hline $55-59$ & $152(17)$ & $135(16)$ & $287(16)$ & \\
\hline $60-80$ & $115(13)$ & $125(15)$ & $240(14)$ & \\
\hline Age at blood draw (years) & & & & 0.35 \\
\hline $40-49$ & $38(4)$ & $30(3)$ & $68(4)$ & \\
\hline $50-59$ & $127(14)$ & $108(13)$ & $235(13)$ & \\
\hline $60-69$ & $362(40)$ & $369(43)$ & $731(42)$ & \\
\hline $70-79$ & $316(35)$ & $281(33)$ & $597(34)$ & \\
\hline$\geq 80$ & $59(7)$ & $70(8)$ & $129(7)$ & \\
\hline Race/Ethnicity & & & & 0.40 \\
\hline White & $812(90)$ & $763(89)$ & $1575(89)$ & \\
\hline Black & $14(2)$ & $7(1)$ & $21(1)$ & \\
\hline Hispanic & $24(3)$ & $32(4)$ & $56(3)$ & \\
\hline Asian/Pacific Islander & $29(3)$ & $32(4)$ & $61(3)$ & \\
\hline Other & $23(3)$ & $24(3)$ & $47(3)$ & \\
\hline Region of Residence (CCR region) & & & & 0.01 \\
\hline 1:Santa Clara Region & $82(9)$ & $83(10)$ & $165(9)$ & \\
\hline 2:Central Region & $99(11)$ & $95(11)$ & $194(11)$ & \\
\hline 3:Sacramento Region & $110(12)$ & $134(16)$ & $244(14)$ & \\
\hline 4:Tri-County Region & $65(7)$ & $62(7)$ & $127(7)$ & \\
\hline 5: Inland Empire Region & $42(5)$ & $25(3)$ & $67(4)$ & \\
\hline 6:North Region & $68(8)$ & $44(5)$ & $112(6)$ & \\
\hline 7:Imperial and San Diego Region & $60(7)$ & $48(6)$ & $108(6)$ & \\
\hline 8:Bay Area Region & $118(13)$ & $99(12)$ & $217(12)$ & \\
\hline 9:Los Angeles County & $173(19)$ & $148(17)$ & $321(18)$ & \\
\hline 10:Orange County & $85(9)$ & $120(14)$ & $205(12)$ & \\
\hline Smoking pack-years & & & & 0.03 \\
\hline Never smoker & $580(64)$ & $573(67)$ & $1153(66)$ & \\
\hline$\leq 10$ & $156(17)$ & $153(18)$ & $309(18)$ & \\
\hline $11-20$ & $47(5)$ & $60(7)$ & $107(6)$ & \\
\hline $21-30$ & $45(5)$ & $23(3)$ & $68(4)$ & \\
\hline$\geq 31$ & $42(5)$ & $27(3)$ & $69(4)$ & \\
\hline Unknown & $32(4)$ & $22(3)$ & $54(3)$ & \\
\hline BMl at baseline $\left(\mathrm{kg} / \mathrm{m}^{2}\right)$ & & & & 0.01 \\
\hline $16.0-24.9$ & $496(55)$ & $519(60)$ & $1015(58)$ & \\
\hline $25.0-29.9$ & $236(26)$ & $203(24)$ & $439(25)$ & \\
\hline $30.0-53.3$ & $153(17)$ & $110(13)$ & $263(15)$ & \\
\hline Unknown & $17(2)$ & $26(3)$ & $43(2)$ & \\
\hline
\end{tabular}


Table 1 Characteristics of study participants $(n=1760)$, by case-control status (Continued)

\begin{tabular}{|c|c|c|c|c|}
\hline \multirow[t]{2}{*}{ Characteristic $^{\mathrm{a}}$} & \multirow{2}{*}{$\begin{array}{l}\text { Case } \\
\text { N (\%) }\end{array}$} & \multirow{2}{*}{$\begin{array}{l}\text { Control } \\
\text { N (\%) }\end{array}$} & \multirow{2}{*}{$\begin{array}{l}\text { All } \\
N(\%)\end{array}$} & \multirow[t]{2}{*}{$p$-value ${ }^{\mathrm{b}}$} \\
\hline & & & & \\
\hline Weight change (Q5-baseline questionnaire) & & & & 0.08 \\
\hline Lost weight & $161(18)$ & $133(16)$ & $294(17)$ & \\
\hline No change (+/-5lbs) & $201(22)$ & $235(27)$ & $436(25)$ & \\
\hline Gained weight & $346(38)$ & $317(37)$ & $663(38)$ & \\
\hline Unknown & $194(22)$ & $173(20)$ & $367(21)$ & \\
\hline Alcohol consumption (g/day) & & & & 0.82 \\
\hline None & $278(31)$ & $250(29)$ & $528(30)$ & \\
\hline$<20$ & $514(57)$ & $507(59)$ & $1021(58)$ & \\
\hline$\geq 20$ & $74(8)$ & $66(8)$ & $140(8)$ & \\
\hline Unknown & $36(4)$ & $35(4)$ & $71(4)$ & \\
\hline Physical activity (hours/week) & & & & 0.20 \\
\hline$<0.50$ & $74(8)$ & $51(6)$ & $125(7)$ & \\
\hline $0.50-3.99$ & $460(51)$ & $438(51)$ & $898(51)$ & \\
\hline$\geq 4.00$ & $367(41)$ & $366(43)$ & $733(42)$ & \\
\hline Unknown & $1(0)$ & $3(0)$ & $4(0)$ & \\
\hline Family history of breast cancer & & & & 0.01 \\
\hline No & $728(81)$ & $737(86)$ & $1465(83)$ & \\
\hline Yes & $136(15)$ & $96(11)$ & $232(13)$ & \\
\hline Unknown & $38(4)$ & $25(3)$ & $63(4)$ & \\
\hline Age at menarche (years) & & & & 0.39 \\
\hline$\leq 11$ & $227(25)$ & $189(22)$ & $416(24)$ & \\
\hline $12-13$ & $500(55)$ & $484(56)$ & $984(56)$ & \\
\hline$\geq 14$ & $166(18)$ & $174(20)$ & $340(19)$ & \\
\hline Unknown/Never & $9(1)$ & $11(1)$ & $20(1)$ & \\
\hline Age at first full-term pregnancy (years) & & & & 0.01 \\
\hline No full-term pregnancy & $233(26)$ & $186(22)$ & $419(24)$ & \\
\hline$\leq 24$ & $209(23)$ & $250(29)$ & $459(26)$ & \\
\hline $25-29$ & $257(28)$ & $257(30)$ & $514(29)$ & \\
\hline$\geq 30$ & $193(21)$ & $151(18)$ & $344(20)$ & \\
\hline Unknown & $10(1)$ & $14(2)$ & $24(1)$ & \\
\hline Breast feeding history (months) & & & & 0.26 \\
\hline Never pregnant & $173(19)$ & $132(15)$ & $305(17)$ & \\
\hline Pregnancy, but no live birth & $58(6)$ & $53(6)$ & $111(6)$ & \\
\hline 0 & $117(13)$ & $125(15)$ & $242(14)$ & \\
\hline$>0$ and $<6$ & $152(17)$ & $130(15)$ & $282(16)$ & \\
\hline $6-11$ & $121(13)$ & $132(15)$ & $253(14)$ & \\
\hline$\geq 12$ & $269(30)$ & $270(31)$ & $539(31)$ & \\
\hline Unknown & $12(1)$ & $16(2)$ & $28(2)$ & \\
\hline Menopausal status at time of blood draw & & & & 0.10 \\
\hline Pre/Peri-menopausal & $43(5)$ & $59(7)$ & $102(6)$ & \\
\hline Postmenopausal & $859(95)$ & $798(93)$ & $1657(94)$ & \\
\hline Unknown & $0(0)$ & $1(0)$ & $1(0)$ & \\
\hline
\end{tabular}

HT use at blood draw ${ }^{c}$ 
Table 1 Characteristics of study participants $(n=1760)$, by case-control status (Continued)

\begin{tabular}{|c|c|c|c|c|}
\hline \multirow[t]{2}{*}{ Characteristic $^{a}$} & \multirow{2}{*}{$\begin{array}{l}\text { Case } \\
\text { N (\%) }\end{array}$} & \multirow{2}{*}{$\begin{array}{l}\text { Control } \\
\text { N (\%) }\end{array}$} & \multirow{2}{*}{$\begin{array}{l}\text { All } \\
\text { N (\%) }\end{array}$} & \multirow[t]{2}{*}{$p$-value } \\
\hline & & & & \\
\hline Never used and postmenopausal & $288(32)$ & $237(28)$ & $525(30)$ & \\
\hline Ever used and postmenopausal & $571(63)$ & $561(65)$ & $1132(64)$ & \\
\hline Pre/Peri-menopausal & $43(5)$ & $59(7)$ & $102(6)$ & \\
\hline Unknown & $0(0)$ & $1(0)$ & $1(0)$ & \\
\hline HT use at baseline & & & & 0.34 \\
\hline Never used & $64(7)$ & $68(8)$ & $132(8)$ & \\
\hline Past HT use & $29(3)$ & $37(4)$ & $66(4)$ & \\
\hline Current estrogen use & $130(14)$ & $143(17)$ & $273(16)$ & \\
\hline Current estrogen and progesterone use & $240(27)$ & $199(23)$ & $439(25)$ & \\
\hline Progesterone use only & $6(1)$ & $10(1)$ & $16(1)$ & \\
\hline Pre-menopausal & $399(44)$ & $367(43)$ & $766(44)$ & \\
\hline Unknown & $34(4)$ & $34(4)$ & $68(4)$ & \\
\hline Age at menopause (years) & & & & $0.22^{d}$ \\
\hline Pre/peri-menopausal & $43(5)$ & $59(7)$ & $102(6)$ & \\
\hline $20-43$ & $143(16)$ & $151(17)$ & $294(17)$ & \\
\hline $44-49$ & $187(21)$ & $139(16)$ & $326(19)$ & \\
\hline $50-51$ & $191(21)$ & $161(19)$ & $352(20)$ & \\
\hline $52-53$ & $112(12)$ & $102(12)$ & $214(12)$ & \\
\hline $54-55$ & $111(12)$ & $119(14)$ & $230(13)$ & \\
\hline $56-65$ & $89(10)$ & $92(11)$ & $181(10)$ & \\
\hline Unknown & $26(3)$ & $35(4)$ & $61(3)$ & \\
\hline Years since onset of menopause & & & & $0.01^{d}$ \\
\hline Pre/peri/unknown menopausal status & $43(5)$ & $60(7)$ & $103(6)$ & \\
\hline $0-9$ & $190(21)$ & $137(16)$ & $327(19)$ & \\
\hline $10-19$ & $29(32)$ & $305(36)$ & $596(34)$ & \\
\hline $20-29$ & $244(27)$ & $196(23)$ & $440(25)$ & \\
\hline $30-69$ & $116(13)$ & $136(16)$ & $252(14)$ & \\
\hline Unknown & $18(2)$ & $24(3)$ & $42(2)$ & \\
\hline Dietary fat & & & & 0.55 \\
\hline Low tertile & $270(30)$ & $274(32)$ & $544(31)$ & \\
\hline Middle tertile & $284(31)$ & $261(30)$ & $545(31)$ & \\
\hline High tertile & $278(31)$ & $269(31)$ & $547(31)$ & \\
\hline Unknown & $70(8)$ & $54(6)$ & $124(7)$ & \\
\hline Pork consumption & & & & 0.01 \\
\hline None & $352(39)$ & $374(44)$ & $726(41)$ & \\
\hline Low (< median) & $193(21)$ & $209(24)$ & $402(23)$ & \\
\hline High ( $\geq$ median) & $287(32)$ & $221(26)$ & $508(29)$ & \\
\hline Unknown & $70(8)$ & $54(6)$ & $124(7)$ & \\
\hline Total red meat consumption & & & & 0.03 \\
\hline Low tertile & $252(28)$ & $291(34)$ & $543(31)$ & \\
\hline Middle tertile & $279(31)$ & $262(31)$ & $541(31)$ & \\
\hline High tertile & $301(33)$ & $25(29)$ & $552(31)$ & \\
\hline Unknown & $70(8)$ & $54(6)$ & $124(7)$ & \\
\hline
\end{tabular}


Table 1 Characteristics of study participants $(n=1760)$, by case-control status (Continued)

\begin{tabular}{|c|c|c|c|c|}
\hline \multirow[t]{2}{*}{ Characteristic $^{a}$} & \multirow{2}{*}{$\begin{array}{l}\text { Case } \\
N(\%)\end{array}$} & \multirow{2}{*}{$\begin{array}{l}\text { Control } \\
\text { N (\%) }\end{array}$} & \multirow{2}{*}{$\begin{array}{l}\text { All } \\
\text { N (\%) }\end{array}$} & \multirow[t]{2}{*}{$p$-value ${ }^{b}$} \\
\hline & & & & \\
\hline Neighborhood socioeconomic status & & & & 0.78 \\
\hline Lowest quintile & $21(2)$ & $25(3)$ & $46(3)$ & \\
\hline 2nd quintile & $69(8)$ & $63(7)$ & $132(8)$ & \\
\hline 3rd quintile & $148(16)$ & $137(16)$ & $285(16)$ & \\
\hline 4th quintile & $260(29)$ & $225(26)$ & $485(28)$ & \\
\hline Highest quintile & $331(37)$ & $334(39)$ & $665(38)$ & \\
\hline Unknown & $73(8)$ & $74(9)$ & $147(8)$ & \\
\hline Neighborhood urbanization & & & & 0.92 \\
\hline Rural/Town & $106(12)$ & $90(10)$ & $196(11)$ & \\
\hline City & $245(27)$ & $230(27)$ & $475(27)$ & \\
\hline Suburban & $440(49)$ & $426(50)$ & $866(49)$ & \\
\hline Metro & $38(4)$ & $38(4)$ & $76(4)$ & \\
\hline Date of blood draw & & & & $<0.01$ \\
\hline 2011 Oct-Dec & $19(2)$ & $147(17)$ & $166(9)$ & \\
\hline 2012 Jan-Jun & $257(28)$ & $151(18)$ & $408(23)$ & \\
\hline 2012 Jul-Dec & $155(17)$ & $69(8)$ & $224(13)$ & \\
\hline 2013 Jan-Jun & $121(13)$ & $142(17)$ & $263(15)$ & \\
\hline 2013 Jul-Dec & $91(10)$ & $81(9)$ & $172(10)$ & \\
\hline 2014 Jan-Jun & $65(7)$ & $67(8)$ & $132(8)$ & \\
\hline 2014 Jul-Dec & $100(11)$ & $108(13)$ & $208(12)$ & \\
\hline 2015 Jan-Jun & $85(9)$ & $82(10)$ & $167(9)$ & \\
\hline 2015 Jul-Aug & $9(1)$ & $11(1)$ & $20(1)$ & \\
\hline Season of blood draw & & & & $<0.01$ \\
\hline Winter & $212(24)$ & $300(35)$ & $512(29)$ & \\
\hline Spring & $275(30)$ & $173(20)$ & $448(25)$ & \\
\hline Summer & $242(27)$ & $125(15)$ & $367(21)$ & \\
\hline Fall & $173(19)$ & $260(30)$ & $433(25)$ & \\
\hline
\end{tabular}

${ }^{a}$ Characteristics assessed at CTS baseline enrollment, unless otherwise noted

${ }^{\mathrm{b}} p$-value for the Chi-square, comparing cases to controls

${ }^{\mathrm{C}} \mathrm{HT}$ use was not characterized for pre- and peri-menopausal women

${ }^{\mathrm{d}}$ Among postmenopausal women at blood draw

below one were observed for PFUnDA and PFHxS. There was also some suggestion of an inverse association for PFOS among those with ER-/PR- tumors $(p=0.06)$. All other analyses conducted among the a priori selected subsets of interest yielded results similar to those observed in the full study population (data not shown).

\section{Discussion}

Overall the findings from this study do not provide evidence that concentrations of PFASs measured in serum collected after diagnosis are related to breast cancer risk in this population of middle-aged and older California women. Our results are consistent with the null findings reported in a pair of C8 Science Panel Studies conducted among Ohio and West Virginia residents with known drinking water PFOA contamination $[49,50]$ and from a Danish population-based nested case-control study [51, 52] but stand in contrast to elevated risks reported from a small case-control study of Greenlandic Inuits [53-55]. Although both the Danish and Inuit study reported increased breast cancer risk associated with PFOSA [51], we could not evaluate risk for PFOSA due to the low frequency of detection in our study population. The inconsistency in findings across this small body of epidemiologic research may be a reflection of differing exposure profiles and host susceptibilities of the study populations considered, as well as features of study design.

The significantly elevated breast cancer risks found among the Greenlandic Inuits [53-55] may be a function of both their uniquely high PFAS exposures and potentially 
Table 2 Serum Concentration of PFAS (ng/mL) among breast cancer cases and controls

\begin{tabular}{|c|c|c|c|c|c|c|c|c|}
\hline & $\mathrm{n}$ & DF & LOD & Mean $^{b}$ & Median $^{\mathrm{b}}$ & $\operatorname{Min}^{\mathrm{b}}$ & $\operatorname{Max}{ }^{b}$ & $p$-value \\
\hline \multicolumn{9}{|c|}{ PFOA (Perfluorooctanoic acid) } \\
\hline Cases & 902 & 99.8 & 0.073 & 2.744 & 2.350 & 0.042 & 39.100 & \\
\hline Controls & 858 & 100.0 & 0.077 & 2.938 & 2.475 & 0.096 & 20.200 & 0.12 \\
\hline \multicolumn{9}{|c|}{ PFNA (Perfluorononanoic acid) } \\
\hline Cases & $871^{a}$ & 99.0 & 0.029 & 0.987 & 0.850 & 0.017 & 7.310 & \\
\hline Controls & 849 & 98.8 & 0.032 & 1.036 & 0.846 & 0.017 & 10.400 & 0.96 \\
\hline \multicolumn{9}{|c|}{ PFUnDA (Perfluoroundecanoic acid) } \\
\hline Cases & 902 & 94.5 & 0.016 & 0.155 & 0.122 & 0.007 & 1.030 & \\
\hline Controls & 858 & 95.5 & 0.018 & 0.163 & 0.128 & 0.007 & 1.310 & 0.22 \\
\hline \multicolumn{9}{|c|}{ PFHxS (Perfluorohexane sulfonic acid } \\
\hline Cases & 902 & 99.8 & 0.022 & 2.217 & 1.515 & 0.011 & 40.700 & \\
\hline Controls & 858 & 99.9 & 0.018 & 2.242 & 1.605 & 0.011 & 21.800 & 0.16 \\
\hline \multicolumn{9}{|c|}{ PFOS (Perfluorooctane sulfonic acid) } \\
\hline Cases & 902 & 99.8 & 0.063 & 8.021 & 6.695 & 0.046 & 39.400 & \\
\hline Controls & 858 & 99.4 & 0.078 & 8.320 & 6.950 & 0.046 & 99.800 & 0.14 \\
\hline \multicolumn{9}{|c|}{ MeFOSAA (2-(N-Methyl-perfluorooctane sulfonamido) acetic acid) } \\
\hline Cases & 902 & 94.4 & 0.018 & 0.301 & 0.152 & 0.007 & 4.000 & \\
\hline Controls & 858 & 94.8 & 0.019 & 0.331 & 0.173 & 0.006 & 8.370 & 0.04 \\
\hline
\end{tabular}

EtFOSSA (2-(N-Ethyl-perfluorooctane sulfonamido) acetic acid)

$\begin{array}{llll}\text { Cases } & 902 & 68.4 & 0.013 \\ \text { Controls } & 858 & 71.3 & 0.015\end{array}$

PFBS (Perfluorobutane sulfonic acid)

$\begin{array}{llll}\text { Cases } & 138^{\mathrm{a}} & 8.0 & 0.053 \\ \text { Controls } & 294 & 17.7 & 0.043\end{array}$

PFDA (Perfluorodecanoic acid)

$\begin{array}{llll}\text { Cases } & 902 & 87.3 & 0.061 \\ \text { Controls } & 858 & 88.7 & 0.053\end{array}$

PFDoDA (Perfluorododeconic acid)

$\begin{array}{llll}\text { Cases } & 880^{\mathrm{a}} & 8.6 & 0.096 \\ \text { Controls } & 858 & 7.5 & 0.099\end{array}$

PFHpA (Perfluoroheptanoic acid)

$\begin{array}{llll}\text { Cases } & 902 & 64.3 & 0.028 \\ \text { Controls } & 858 & 62.6 & 0.030\end{array}$

PFOSA (Perfluorooctane sulfonamide)

$\begin{array}{llll}\text { Cases } & 672^{\mathrm{a}} & 51.6 & 0.023 \\ \text { Controls } & 775 & 65.2 & 0.019\end{array}$

$D F$ detection frequency, $L O D$ average limit of detection, Min minimum, Max maximum

a Due to laboratory failure 40 PFNA, 1328 PFBS, 22 PFDoA, and 313 PFOSA sample concentrations were not reported

${ }^{b}$ Descriptive statistics were not generated for the PFASs excluded from analysis due to DF $<95 \%$

${ }^{c} p$-value from the Wilcoxon rank sum test for differences in medians by case-control status

greater genetic susceptibility to risks associated with those exposures. The serum levels of some PFASs reported in the Inuits are especially high compared to the levels in our population, as well as those reported in other areas of the world [68]. Specifically, in comparison to the median levels in our controls, the median levels in the Inuit controls were approximately $162 \%, 200 \%$, and $1700 \%$ higher for PFOS, PFNA, and PFUnDA, respectively (Additional file 1: Table $\mathrm{S} 1)$. Thus the elevated breast cancer risks observed for PFOS among the Inuits but not in our study may be a reflection of their higher exposures. However, for PFOA and PFHxS (two PFASs for which elevated breast cancer risks 
Table 3 Invasive breast cancer risk associated with serum PFAS concentrations among 1760 study participants

\begin{tabular}{|c|c|c|c|c|c|}
\hline PFAS Serum Concentration & \# cases & $\begin{array}{l}\text { Crude }^{a} \\
\text { OR (95\% Cl) }\end{array}$ & $p$-value ${ }^{b}$ & $\begin{array}{l}\text { Adjusted }^{c} \\
\text { OR (95\% Cl) }\end{array}$ & $p$-value \\
\hline \multicolumn{6}{|l|}{ PFOA } \\
\hline Low & 331 & 1.00 (ref) & 0.32 & 1.00 (ref) & 0.54 \\
\hline Medium & 298 & $0.904(0.718,1.139)$ & & $0.901(0.705,1.152)$ & \\
\hline High & 273 & $0.888(0.698,1.130)$ & & $0.925(0.715,1.197)$ & \\
\hline $\log [$ PFOA, ng/mL] & 902 & $0.718(0.499,1.034)$ & 0.08 & $0.733(0.496,1.081)$ & 0.11 \\
\hline \multicolumn{6}{|l|}{ PFNA } \\
\hline Low & 285 & 1.00 (ref) & 0.48 & 1.00 (ref) & 0.79 \\
\hline Medium & 297 & $1.083(0.855,1.371)$ & & $1.043(0.808,1.345)$ & \\
\hline High & 289 & $1.091(0.859,1.384)$ & & $1.037(0.798,1.348)$ & \\
\hline $\log [$ PFNA, ng/mL] & $871^{d}$ & $0.990(0.724,1.354)$ & 0.95 & $0.880(0.624,1.240)$ & 0.46 \\
\hline \multicolumn{6}{|l|}{ PFUnDA } \\
\hline Low & 312 & 1.00 (ref) & 0.11 & 1.00 (ref) & 0.08 \\
\hline Medium & 335 & $1.080(0.859,1.357)$ & & $1.106(0.866,1.413)$ & \\
\hline High & 255 & $0.817(0.642,1.039)$ & & $0.786(0.605,1.020)$ & \\
\hline $\log [$ PFUnDA, ng/mL] & 902 & $0.855(0.668,1.094)$ & 0.22 & $0.855(0.653,1.118)$ & 0.25 \\
\hline \multicolumn{6}{|l|}{$\mathrm{PFH} \times \mathrm{S}$} \\
\hline Low & 363 & 1.00 (ref) & 0.02 & 1.00 (ref) & 0.08 \\
\hline Medium & 263 & $0.734(0.580,0.930)$ & & $0.798(0.621,1.025)$ & \\
\hline High & 276 & $0.762(0.598,0.970)$ & & $0.801(0.619,1.035)$ & \\
\hline $\log [\mathrm{PFH} \times \mathrm{S}, \mathrm{ng} / \mathrm{mL}]$ & 902 & $0.780(0.583,1.042)$ & 0.09 & $0.811(0.596,1.104)$ & 0.18 \\
\hline \multicolumn{6}{|l|}{ PFOS } \\
\hline Low & 318 & 1.00 (ref) & 0.60 & 1.00 (ref) & 0.41 \\
\hline Medium & 297 & $0.955(0.757,1.206)$ & & $0.883(0.691,1.129)$ & \\
\hline High & 287 & $0.938(0.738,1.193)$ & & $0.898(0.695,1.161)$ & \\
\hline $\log [$ PFOS, ng/mL] & 902 & $1.011(0.754,1.356)$ & 0.94 & $0.934(0.683,1.277)$ & 0.67 \\
\hline \multicolumn{6}{|l|}{ MeFOSAA } \\
\hline Low & 349 & 1.00 (ref) & 0.07 & 1.00 (ref) & 0.29 \\
\hline Medium & 278 & $0.804(0.638,1.014)$ & & $0.847(0.663,1.083)$ & \\
\hline High & 275 & $0.812(0.644,1.026)$ & & $0.877(0.682,1.126)$ & \\
\hline $\log [\mathrm{MeFOSAA}, \mathrm{ng} / \mathrm{mL}]$ & 902 & $0.873(0.715,1.066)$ & 0.18 & $0.960(0.774,1.191)$ & 0.71 \\
\hline
\end{tabular}

$O R$ odds ratio, $\mathrm{Cl}$ confidence interval

${ }^{a}$ Crude ORs adjusted for matching design variables of age at baseline enrollment, race/ethnicity, and region of residence

${ }^{b}$ for the categorical analysis, the $p$-values represent a test for linear trend with tertiles of PFAS modeled as a 3-level ordinal variable; for the continuous PFAS term, the $p$-value represents the $p$-value of the Wald-statistic for the $\beta$-coefficient for the PFAS modeled as a continuous term

'ORs adjusted for age at baseline enrollment, race/ethnicity, region of residence, date of blood draw, date of blood draw ${ }^{2}$, season of blood draw, total smoking pack-years, BMI, family history of breast cancer, age at first full-term pregnancy, menopausal status at blood draw, and pork consumption

${ }^{\mathrm{d}}$ PFNA excludes $n=40$ with non-reportable serum value, (cases: $n=31$; controls: $n=9$ )

were observed in the Inuits and not in our study) the median levels in the Inuits were approximately 59 and $67 \%$ lower (respectively) than in our study. Furthermore, PFOA concentrations in the Danish study, and particularly the C8 Science Panel Study, both of which reported no breast cancer risks, were much higher than in the Inuit. Thus, it seems unlikely that the positive findings among the Inuits are solely a function of high PFAS exposures. This Inuit population, however, also has notably high body burdens of a number of other persistent organic pollutants (POPs) that have been suspected to play a role in breast cancer risk, including polychlorinated biphenyl ethers (PCBs) [69] of which some are much higher than in our study population. It is possible that uncontrolled confounding for exposures to other carcinogenic or xenoestrogenic compounds such as PCBs could be driving the positive PFAS effects reported in the Inuits. In fact, increased risks of breast cancer were also reported in the Inuit study for a number of PCBs [55].

The increased breast cancer risks observed among the Greenlandic Inuits may also reflect greater susceptibility 
Table 4 Invasive breast cancer risk associated with PFAS concentrations, by menopausal status ${ }^{2}$

\begin{tabular}{|c|c|c|c|c|c|c|c|c|c|c|}
\hline \multirow[t]{3}{*}{$\begin{array}{l}\text { PFAS Serum } \\
\text { Concentration }\end{array}$} & \multicolumn{5}{|c|}{$\begin{array}{l}\text { Postmenopausal } \\
(n=1657)\end{array}$} & \multicolumn{5}{|c|}{$\begin{array}{l}\text { Pre- or Peri-menopausal } \\
(n=102)\end{array}$} \\
\hline & \multirow[t]{2}{*}{ \# cases } & \multicolumn{2}{|l|}{$\begin{array}{l}\text { Crude Odds } \\
\text { Ratio (OR) }{ }^{\text {b }}\end{array}$} & \multicolumn{2}{|l|}{$\begin{array}{l}\text { Adjusted Odds } \\
\text { Ratio }(O R)^{d}\end{array}$} & \multirow[t]{2}{*}{ \# cases } & \multicolumn{2}{|l|}{$\begin{array}{l}\text { Crude Odds } \\
\text { Ratio (OR) }{ }^{\text {b }}\end{array}$} & \multicolumn{2}{|l|}{$\begin{array}{l}\text { Adjusted Odds } \\
\text { Ratio (OR) }{ }^{\text {e }}\end{array}$} \\
\hline & & OR $(95 \% \mathrm{Cl})$ & $p$-value ${ }^{c}$ & $\mathrm{OR}(95 \% \mathrm{Cl})$ & $p$-value ${ }^{c}$ & & OR $(95 \% \mathrm{Cl})$ & $p$-value ${ }^{c}$ & $\mathrm{OR}(95 \% \mathrm{Cl})$ & $p$-value \\
\hline \multicolumn{11}{|l|}{$\overline{\mathrm{PFOA}}$} \\
\hline Low & 306 & 1.00 & 0.27 & 1.00 & 0.49 & 25 & 1.00 & 0.77 & 1.00 & 0.62 \\
\hline Medium & 287 & $\begin{array}{l}0.868 \\
(0.682,1.103)\end{array}$ & & $\begin{array}{l}0.889 \\
(0.689,1.147)\end{array}$ & & 11 & $\begin{array}{l}0.881 \\
(0.318,2.443)\end{array}$ & & $\begin{array}{l}0.888 \\
(0.239,3.302)\end{array}$ & \\
\hline High & 266 & $\begin{array}{l}0.870 \\
(0.679,1.116)\end{array}$ & & $\begin{array}{l}0.912 \\
(0.699,1.189)\end{array}$ & & 7 & $\begin{array}{l}0.859 \\
(0.264,2.796)\end{array}$ & & $\begin{array}{l}0.669 \\
(0.143,3.119)\end{array}$ & \\
\hline $\log [$ PFOA, ng/mL] & 859 & $\begin{array}{l}0.696 \\
(0.475,1.019)\end{array}$ & 0.06 & $\begin{array}{l}0.715 \\
(0.476,1.073)\end{array}$ & 0.11 & 43 & $\begin{array}{l}0.352 \\
(0.077,1.613)\end{array}$ & 0.18 & $\begin{array}{l}0.177 \\
(0.023,1.342)\end{array}$ & 0.09 \\
\hline \multicolumn{11}{|l|}{ PFNA } \\
\hline Low & 266 & 1.00 & 0.92 & 1.00 & 0.71 & 19 & 1.00 & 0.06 & 1.00 & 0.06 \\
\hline Medium & 286 & $\begin{array}{l}1.016 \\
(0.795,1.298)\end{array}$ & & $\begin{array}{l}0.978 \\
(0.751,1.275)\end{array}$ & & 11 & $\begin{array}{l}1.373 \\
(0.469,4.017)\end{array}$ & & $\begin{array}{l}1.373 \\
(0.469,4.017)\end{array}$ & \\
\hline High & 277 & $\begin{array}{l}1.013 \\
(0.791,1.297)\end{array}$ & & $\begin{array}{l}0.949 \\
(0.723,1.247)\end{array}$ & & 12 & $\begin{array}{l}3.117 \\
(0.975,9.964)\end{array}$ & & $\begin{array}{l}3.117 \\
(0.975,9.964)\end{array}$ & \\
\hline $\log [$ PFNA, ng/mL] & $829^{f}$ & $\begin{array}{l}0.941 \\
(0.681,1.301)\end{array}$ & 0.71 & $\begin{array}{l}0.837 \\
(0.587,1.195)\end{array}$ & 0.33 & $42^{f}$ & $\begin{array}{l}1.360 \\
(0.293,6.309)\end{array}$ & 0.69 & $\begin{array}{l}1.360 \\
(0.293,6.309)\end{array}$ & 0.69 \\
\hline \multicolumn{11}{|l|}{ PFUnDA } \\
\hline Low & 290 & 1.00 & 0.14 & 1.00 & 0.08 & 22 & 1.00 & 0.20 & 1.00 & 0.04 \\
\hline Medium & 318 & $\begin{array}{l}1.096 \\
(0.863,1.391)\end{array}$ & & $\begin{array}{l}1.100 \\
(0.853,1.419)\end{array}$ & & 17 & $\begin{array}{l}0.847 \\
(0.341,2.101)\end{array}$ & & $\begin{array}{l}0.476 \\
(0.157,1.445)\end{array}$ & \\
\hline High & 251 & $\begin{array}{l}0.828 \\
(0.646,1.062)\end{array}$ & & $\begin{array}{l}0.784 \\
(0.600,1.024)\end{array}$ & & 4 & $\begin{array}{l}0.365 \\
(0.090,1.469)\end{array}$ & & $\begin{array}{l}0.179 \\
(0.033,0.970)\end{array}$ & \\
\hline $\log [$ PFUnDA, ng/mL] & 859 & $\begin{array}{l}0.838 \\
(0.649,1.082)\end{array}$ & 0.18 & $\begin{array}{l}0.827 \\
(0.628,1.091)\end{array}$ & 0.18 & 43 & $\begin{array}{l}0.769 \\
(0.251,2.354)\end{array}$ & 0.65 & $\begin{array}{l}0.377 \\
(0.095,1.494)\end{array}$ & 0.16 \\
\hline \multicolumn{11}{|l|}{ PFH $\times S$} \\
\hline Low & 334 & 1.00 & 0.01 & 1.00 & 0.05 & 29 & 1.00 & 0.92 & 1.00 & 0.18 \\
\hline Medium & 253 & $\begin{array}{l}0.700 \\
(0.548,0.893)\end{array}$ & & $\begin{array}{l}0.762 \\
(0.589,0.986)\end{array}$ & & 10 & $\begin{array}{l}1.102 \\
(0.381,3.192)\end{array}$ & & $0.659(0.184,2.359)$ & \\
\hline High & 272 & $\begin{array}{l}0.733 \\
(0.573,0.939)\end{array}$ & & $\begin{array}{l}0.769 \\
(0.592,0.999)\end{array}$ & & 4 & $\begin{array}{l}0.817 \\
(0.170,3.936)\end{array}$ & & $0.286(0.043,1.892)$ & \\
\hline $\log [\mathrm{PFHxS}, \mathrm{ng} / \mathrm{mL}]$ & 859 & $\begin{array}{l}0.728 \\
(0.539,0.985)\end{array}$ & 0.04 & $\begin{array}{l}0.759 \\
(0.511,1.044)\end{array}$ & 0.09 & 43 & $\begin{array}{l}1.095 \\
(0.317,3.788)\end{array}$ & 0.89 & $0.577(0.143,2.325)$ & 0.44 \\
\hline \multicolumn{11}{|l|}{ PFOS } \\
\hline Low & 293 & 1.00 & 0.35 & 1.00 & 0.26 & 25 & 1.00 & 0.23 & 1.00 & 0.57 \\
\hline Medium & 284 & $\begin{array}{l}0.903 \\
(0.708,1.150)\end{array}$ & & $\begin{array}{l}0.843 \\
(0.653,1.088)\end{array}$ & & 13 & $\begin{array}{l}1.620 \\
(0.559,4.691)\end{array}$ & & $\begin{array}{l}1.796 \\
(0.493,6.546)\end{array}$ & \\
\hline High & 282 & $\begin{array}{l}0.888 \\
(0.694,1.135)\end{array}$ & & $\begin{array}{l}0.860 \\
(0.661,1.118)\end{array}$ & & 5 & $\begin{array}{l}2.345 \\
(0.439,12.536)\end{array}$ & & $\begin{array}{l}1.208 \\
(0.163,8.944)\end{array}$ & \\
\hline $\log [$ PFOS, ng/mL] & 859 & $\begin{array}{l}0.951 \\
(0.703,1.288)\end{array}$ & 0.75 & $\begin{array}{l}0.885 \\
(0.641,1.223)\end{array}$ & 0.46 & 43 & $\begin{array}{l}2.006 \\
(0.472,8.524)\end{array}$ & 0.35 & $\begin{array}{l}0.900 \\
(0.166,4.876)\end{array}$ & 0.90 \\
\hline \multicolumn{11}{|l|}{ MeFOSAA } \\
\hline Low & 329 & 1.00 & 0.09 & 1.00 & 0.31 & 20 & 1.00 & 0.99 & 1.00 & 0.73 \\
\hline Medium & 265 & $\begin{array}{l}0.829 \\
(0.652,1.055)\end{array}$ & & $\begin{array}{l}0.865 \\
(0.670,1.116)\end{array}$ & & 13 & $\begin{array}{l}0.539 \\
(0.181,1.609)\end{array}$ & & $\begin{array}{l}0.332 \\
(0.092,1.200)\end{array}$ & \\
\hline High & 265 & $\begin{array}{l}0.816 \\
(0.642,1.037)\end{array}$ & & $\begin{array}{l}0.877 \\
(0.677,1.135)\end{array}$ & & 10 & $\begin{array}{l}1.033 \\
(0.322,3.313)\end{array}$ & & $\begin{array}{l}0.876 \\
(0.223,3.445)\end{array}$ & \\
\hline $\log [\mathrm{MeFOSAA}, \mathrm{ng} / \mathrm{mL}]$ & 859 & 0.854 & 0.13 & 0.929 & 0.52 & 43 & 1.567 & 0.36 & 1.414 & 0.54 \\
\hline
\end{tabular}


Table 4 Invasive breast cancer risk associated with PFAS concentrations, by menopausal status ${ }^{a}$ (Continued)

\begin{tabular}{|c|c|c|c|c|c|c|c|c|c|c|}
\hline \multirow[t]{3}{*}{$\begin{array}{l}\text { PFAS Serum } \\
\text { Concentration }\end{array}$} & \multicolumn{5}{|c|}{$\begin{array}{l}\text { Postmenopausal } \\
(n=1657)\end{array}$} & \multicolumn{5}{|c|}{$\begin{array}{l}\text { Pre- or Peri-menopausal } \\
(n=102)\end{array}$} \\
\hline & \multirow[t]{2}{*}{ \# cases } & \multicolumn{2}{|l|}{$\begin{array}{l}\text { Crude Odds } \\
\text { Ratio (OR) }\end{array}$} & \multicolumn{2}{|c|}{$\begin{array}{l}\text { Adjusted Odds } \\
\text { Ratio }(\mathrm{OR}) \mathrm{d}\end{array}$} & \multirow[t]{2}{*}{ \# cases } & \multicolumn{2}{|l|}{$\begin{array}{l}\text { Crude Odds } \\
\text { Ratio (OR) }\end{array}$} & \multicolumn{2}{|l|}{$\begin{array}{l}\text { Adjusted Odds } \\
\text { Ratio }(\mathrm{OR})^{\mathrm{e}}\end{array}$} \\
\hline & & $\overline{\mathrm{OR}}(95 \% \mathrm{Cl})$ & $\overline{p \text {-value }}{ }^{c}$ & $\overline{\mathrm{OR}}(95 \% \mathrm{Cl})$ & $\overline{p \text {-value }}{ }^{c}$ & & $\overline{\mathrm{OR}}(95 \% \mathrm{Cl})$ & $\overline{p \text {-value }}{ }^{c}$ & $\overline{\mathrm{OR}}(95 \% \mathrm{Cl})$ & $p$-value \\
\hline & & $(0.695,1.049)$ & & $(0.743,1.161)$ & & & $(0.601,4.089)$ & & $(0.465,4.293)$ & \\
\hline
\end{tabular}

OR odds ratio, $\mathrm{Cl}$ confidence interval

${ }^{a}$ Based on menopausal status at blood draw; excludes 1 participant for whom menopausal status was unknown

b Crude ORs adjusted for design variables of age at baseline enrollment, race/ethnicity, region of residence

${ }^{c}$ For the categorical analysis, the $p$-values represent a test for linear trend with tertiles of PFAS modeled as a 3-level ordinal variable; for the continuous PFAS

term, the $p$-value represents the $p$-value of the Wald-statistic for the $\beta$-coefficient for the PFAS modeled as a continuous term

${ }^{d}$ For PFOA, PFNA, PFOS, MeFOSAA, covariates included in the fully-adjusted model were:

age at baseline enrollment, race/ethnicity, region of residence, blood draw date, blood draw date ${ }^{2}$, season of blood draw, total pack-years smoking, BMI, family history of breast cancer, age at first full-term pregnancy, pork consumption. For PFUnDA and PFHxS covariates included in adjusted models were: age at baseline enrollment, race/ethnicity, region of residence, blood draw date, blood draw date ${ }^{2}$, season of blood draw, total pack-years smoking, family history of breast cancer, age at first full-term pregnancy, pork consumption

${ }^{\mathrm{e}}$ For PFOA covariates included in all fully-adjusted models were: age at baseline enrollment (20-39/40-80+), race/ethnicity (white/non-white), region of residence, blood draw date, blood draw date ${ }^{2}$, season of blood draw, dietary fat, and total red meat consumption. For PFOS, PFUnDA, PFHxS, and MeFOSAA covariates included in all fully-adjusted models were: age at baseline enrollment (20-39/40-80+), race/ethnicity (white/non-white), region of residence, season of blood draw, and total red meat consumption. For PFNA covariates included in all fully-adjusted models were: age at baseline enrollment (20-39/40-80+), race/ethnicity (white/non-white), and region of residence

f PFNA: Postmenopausal excludes $n=39$ with non-reportable serum value, (cases: $n=30$; controls: $n=9$ ); Pre-/Peri-menopausal exclude $n=1$ case with nonreportable serum value

to PFAS exposures. Analyses of genetic polymorphisms in genes that are involved in estrogen metabolism and estrogen biosynthesis (e.g. CYP, COMT) indicated polymorphic frequencies in this Inuit population that are distinctly different than those reported in Euro-Caucasian populations that have been the focus of the other breast cancer studies to date [54]. While the Inuit study was under-powered to statistically evaluate gene-environment interactions, there was some indication that the risks associated with PFASs and other POPs were more pronounced in those with specific polymorphisms in the targeted genes of interest [54]. This is an area of inquiry that deserves additional attention.

Another potential explanation for the disparate findings across the small body of studies conducted to date could be the variability in methodologic approaches, especially with regards to exposure assessment. Our approach for estimating PFAS exposure was more similar to that used in the Greenlandic Inuit study but our findings are more consistent with the null results reported from the Danish $[51,52]$ and C8 Science Panel $[49,50]$ studies which used markedly different exposure ascertainment methods. Both our study and the Inuit study relied on serum concentrations of PFAS measured in blood that was collected after diagnosis. While single measures of PFAS in serum are generally regarded as reasonable measures of chronic exposures [68], the degree to which PFAS concentrations may be affected by hormonal or other physiologic changes associated with the onset of and/or sequelae and treatment of breast cancer is not known. Thus, the possibility of reverse causation bias in our study and the Inuit study cannot be fully discounted.

An important distinction between our study and the Inuit study is that while both relied on blood collected post-diagnosis, in the Inuit study the blood was collected prior to treatment while in our study this was not the case. To our knowledge, the effects of breast cancer treatment, if any, on levels of serum PFAS have not been explored and remain unknown. If breast cancer treatment causes declines in PFAS levels, this would have limited our ability to detect an increase in breast cancer risk and could have resulted in spurious inverse associations. Unfortunately, complete information on treatment is not available for the CTS cohort.

Exposure assessment in the C8 Science Panel Study, while retrospective, was not based on body burden measurements but rather was derived from complex modeling incorporating information from self-reported residential histories, historical data on PFOA drinking water contamination, occupational histories and a job exposure matrix to estimate cumulative PFOA exposures. While these estimates were validated with serum PFOA levels in a subset of participants, the possibility of exposure misclassification remains.

A unique strength of the Danish study is its reliance on quantitatively-measured values of PFASs in blood samples collected 10 to 15 years prior to breast cancer diagnosis/enrollment. Notably, however, the blood samples, were collected during early pregnancy. This poses some complication in the interpretation of the study's results. Pregnancy-related changes in blood volume and alterations in concentrations of serum albumin [70] to which PFASs bind, may affect PFAS serum levels. Analyses of NHANEs data have suggested lower PFOS levels in pregnant women [71]. Furthermore, the expulsion of the fetus and placenta and blood loss accompanying parturition is considered a major route of PFAS elimination among parous women [72]. Thus, the measurement of PFASs in blood specimens collected during pregnancy 
Table 5 Invasive breast cancer risk associated with serum PFAS, by tumor hormone receptor status ${ }^{a}$

\begin{tabular}{|c|c|c|c|c|c|c|c|c|c|c|}
\hline \multirow[t]{3}{*}{$\begin{array}{l}\text { PFAS Serum } \\
\text { Concentration }\end{array}$} & \multicolumn{5}{|c|}{$\begin{array}{l}\text { ER+ or PR+ } \\
(n=1601)\end{array}$} & \multicolumn{5}{|c|}{$\begin{array}{l}\text { ER- and PR- } \\
(n=965)\end{array}$} \\
\hline & \multirow[t]{2}{*}{ \# cases } & \multicolumn{2}{|l|}{$\begin{array}{l}\text { Crude Odds } \\
\text { Ratio (OR) }{ }^{\text {b }}\end{array}$} & \multicolumn{2}{|l|}{$\begin{array}{l}\text { Adjusted Odds } \\
\text { Ratio (OR) }\end{array}$} & \multirow[t]{2}{*}{ \# cases } & \multicolumn{2}{|l|}{$\begin{array}{l}\text { Crude Odds } \\
\text { Ratio (OR) }{ }^{\text {b }}\end{array}$} & \multicolumn{2}{|l|}{$\begin{array}{l}\text { Adjusted Odds } \\
\text { Ratio (OR) }\end{array}$} \\
\hline & & OR $(95 \% \mathrm{Cl})^{\mathrm{b}}$ & $p$-value ${ }^{c}$ & OR $(95 \% \mathrm{CI})^{d}$ & $p$-value ${ }^{c}$ & & $\mathrm{OR}(95 \% \mathrm{Cl})^{\mathrm{b}}$ & $p$-value ${ }^{c}$ & $\mathrm{OR}(95 \% \mathrm{Cl})^{\mathrm{e}}$ & $p$-value \\
\hline \multicolumn{11}{|l|}{$\overline{\mathrm{PFOA}}$} \\
\hline Low & 266 & 1.00 & 0.50 & 1.00 & 0.71 & 43 & 1.00 & 0.38 & 1.00 & 0.39 \\
\hline Medium & 247 & $\begin{array}{l}0.923 \\
(0.723,1.178)\end{array}$ & & $\begin{array}{l}0.918 \\
(0.707,1.191)\end{array}$ & & 35 & $\begin{array}{l}0.824 \\
(0.505,1.346)\end{array}$ & & $\begin{array}{l}0.846 \\
(0.510,1.403)\end{array}$ & \\
\hline High & 230 & $\begin{array}{l}0.917 \\
(0.711,1.182)\end{array}$ & & $\begin{array}{l}0.952 \\
(0.725,1.251)\end{array}$ & & 29 & $\begin{array}{l}0.800 \\
(0.474,1.348)\end{array}$ & & $\begin{array}{l}0.792 \\
(0.460,1.365)\end{array}$ & \\
\hline $\log [$ PFOA, ng/mL] & 743 & $\begin{array}{l}0.781 \\
(0.529,1.151)\end{array}$ & 0.21 & $\begin{array}{l}0.779 \\
(0.513,1.183)\end{array}$ & 0.24 & 107 & $\begin{array}{l}0.523 \\
(0.246,1.111)\end{array}$ & 0.09 & $\begin{array}{l}0.528 \\
(0.239,1.165)\end{array}$ & 0.11 \\
\hline \multicolumn{11}{|l|}{ PFNA } \\
\hline Low & 233 & 1.00 & 0.57 & 1.00 & 0.76 & 33 & 1.00 & 0.43 & 1.00 & 0.51 \\
\hline Medium & 250 & $\begin{array}{l}1.112 \\
(0.868,1.426)\end{array}$ & & $\begin{array}{l}1.034 \\
(0.789,1.354)\end{array}$ & & 34 & $\begin{array}{l}1.107 \\
(0.659,1.859)\end{array}$ & & $\begin{array}{l}1.002 \\
(0.583,1.720)\end{array}$ & \\
\hline High & 234 & $\begin{array}{l}1.076 \\
(0.835,1.387)\end{array}$ & & $\begin{array}{l}0.959 \\
(0.724,1.270)\end{array}$ & & 36 & $\begin{array}{l}1.231 \\
(0.734,2.064)\end{array}$ & & $\begin{array}{l}1.186 \\
(0.690,2.039)\end{array}$ & \\
\hline $\log [$ PFNA, ng/mL] & $717^{f}$ & $\begin{array}{l}1.096 \\
(0.779,1.543)\end{array}$ & 0.60 & $\begin{array}{l}0.924 \\
(0.634,1.346)\end{array}$ & 0.68 & $103^{f}$ & $\begin{array}{l}0.783 \\
(0.416,1.475)\end{array}$ & 0.45 & $\begin{array}{l}0.701 \\
(0.357,1.375)\end{array}$ & 0.30 \\
\hline \multicolumn{11}{|l|}{ PFUnDA } \\
\hline Low & 249 & 1.00 & 0.38 & 1.00 & 0.22 & 43 & 1.00 & 0.03 & 1.00 & 0.05 \\
\hline Medium & 274 & $\begin{array}{l}1.116 \\
(0.876,1.422)\end{array}$ & & $\begin{array}{l}1.125 \\
(0.866,1.461)\end{array}$ & & 41 & $\begin{array}{l}0.947 \\
(0.591,1.518)\end{array}$ & & $\begin{array}{l}1.007 \\
(0.614,1.651)\end{array}$ & \\
\hline High & 220 & $\begin{array}{l}0.889 \\
(0.689,1.146)\end{array}$ & & $\begin{array}{l}0.838 \\
(0.636,1.106)\end{array}$ & & 23 & $\begin{array}{l}0.522 \\
(0.298,0.912)\end{array}$ & & $\begin{array}{l}0.550 \\
(0.307,0.985)\end{array}$ & \\
\hline $\log [$ PFUnDA, ng/mL] & 743 & $\begin{array}{l}0.922 \\
(0.710,1.196)\end{array}$ & 0.54 & $\begin{array}{l}0.892 \\
(0.671,1.185)\end{array}$ & 0.43 & 107 & $\begin{array}{l}0.661 \\
(0.386,1.133)\end{array}$ & 0.13 & $\begin{array}{l}0.665 \\
(0.375,1.179)\end{array}$ & 0.16 \\
\hline \multicolumn{11}{|l|}{ PFHXS } \\
\hline Low & 287 & 1.00 & 0.10 & 1.00 & 0.25 & 54 & 1.00 & 0.02 & 1.00 & 0.03 \\
\hline Medium & 219 & $\begin{array}{l}0.763 \\
(0.594,0.980)\end{array}$ & & $\begin{array}{l}0.830 \\
(0.635,1.084)\end{array}$ & & 28 & $\begin{array}{l}0.582 \\
(0.352,0.962)\end{array}$ & & $\begin{array}{l}0.587 \\
(0.350,0.985)\end{array}$ & \\
\hline High & 237 & $\begin{array}{l}0.812 \\
(0.629,1.048)\end{array}$ & & $\begin{array}{l}0.855 \\
(0.651,1.122)\end{array}$ & & 25 & $\begin{array}{l}0.558 \\
(0.326,0.954)\end{array}$ & & $\begin{array}{l}0.567 \\
(0.326,0.985)\end{array}$ & \\
\hline $\log [\mathrm{PFHxS}, \mathrm{ng} / \mathrm{mL}]$ & 743 & $\begin{array}{l}0.874 \\
(0.640,1.193)\end{array}$ & 0.40 & $\begin{array}{l}0.915 \\
(0.655,1.279)\end{array}$ & 0.60 & 107 & $\begin{array}{l}0.449 \\
(0.250,0.807)\end{array}$ & 0.01 & $\begin{array}{l}0.446 \\
(0.243,0.821)\end{array}$ & 0.01 \\
\hline \multicolumn{11}{|l|}{ PFOS } \\
\hline Low & 250 & 1.00 & 0.83 & 1.00 & 0.81 & 47 & 1.00 & 0.09 & 1.00 & 0.06 \\
\hline Medium & 247 & $\begin{array}{l}1.020 \\
(0.796,1.305)\end{array}$ & & $\begin{array}{l}0.937 \\
(0.721,1.218)\end{array}$ & & 32 & $\begin{array}{l}0.697 \\
(0.427,1.139)\end{array}$ & & $\begin{array}{l}0.628 \\
(0.378,1.041)\end{array}$ & \\
\hline High & 246 & $\begin{array}{l}1.029 \\
(0.798,1.327)\end{array}$ & & $\begin{array}{l}0.967 \\
(0.737,1.270)\end{array}$ & & 28 & $\begin{array}{l}0.652 \\
(0.387,1.100)\end{array}$ & & $\begin{array}{l}0.615 \\
(0.357,1.059)\end{array}$ & \\
\hline $\log [$ PFOS, ng/mL] & 743 & $\begin{array}{l}1.177 \\
(0.852,1.626)\end{array}$ & 0.32 & $\begin{array}{l}1.054 \\
(0.744,1.493)\end{array}$ & 0.77 & 107 & $0.645(0.375,1.109)$ & 0.11 & $\begin{array}{l}0.573 \\
(0.323,1.016)\end{array}$ & 0.06 \\
\hline \multicolumn{11}{|l|}{ MeFOSAA } \\
\hline Low & 289 & 1.00 & 0.08 & 1.00 & 0.23 & 36 & 1.00 & 0.91 & 1.00 & 0.89 \\
\hline Medium & 228 & $\begin{array}{l}0.802 \\
(0.628,1.023)\end{array}$ & & $\begin{array}{l}0.855 \\
(0.660,1.109)\end{array}$ & & 39 & $1.106(0.675,1.814)$ & & $\begin{array}{l}1.124 \\
(0.674,1.877)\end{array}$ & \\
\hline High & 226 & $\begin{array}{l}0.806 \\
(0.630,1.030)\end{array}$ & & $\begin{array}{l}0.854 \\
(0.655,1.113)\end{array}$ & & 32 & $\begin{array}{l}0.968 \\
(0.575,1.630)\end{array}$ & & $\begin{array}{l}0.960 \\
(0.557,1.654)\end{array}$ & \\
\hline $\log [\mathrm{MeFOSAA}, \mathrm{ng} / \mathrm{mL}]$ & 743 & 0.864 & 0.18 & 0.932 & 0.55 & 107 & 0.983 & 0.94 & 0.996 & 0.98 \\
\hline
\end{tabular}


Table 5 Invasive breast cancer risk associated with serum PFAS, by tumor hormone receptor status ${ }^{\text {a }}$ (Continued)

\begin{tabular}{|c|c|c|c|c|c|c|c|c|c|}
\hline \multirow[t]{3}{*}{$\begin{array}{l}\text { PFAS Serum } \\
\text { Concentration }\end{array}$} & \multicolumn{5}{|c|}{$\begin{array}{l}\text { ER+ or PR+ } \\
(n=1601)\end{array}$} & \multicolumn{4}{|c|}{$\begin{array}{l}\text { ER- and PR- } \\
(n=965)\end{array}$} \\
\hline & \multirow[t]{2}{*}{ \# cases } & \multicolumn{2}{|l|}{$\begin{array}{l}\text { Crude Odds } \\
\text { Ratio (OR) }\end{array}$} & \multicolumn{2}{|l|}{$\begin{array}{l}\text { Adjusted Odds } \\
\text { Ratio }(\mathrm{OR}) \mathrm{d}\end{array}$} & \multirow[t]{2}{*}{ \# cases } & \multicolumn{2}{|l|}{$\begin{array}{l}\text { Crude Odds } \\
\text { Ratio (OR) }{ }^{b}\end{array}$} & $\begin{array}{l}\text { Adjusted Odds } \\
\text { Ratio }(\mathrm{OR}) \mathrm{e}^{\mathrm{e}}\end{array}$ \\
\hline & & $\overline{\text { OR }(95 \% ~ C l)^{b}}$ & $\overline{p \text {-value }}{ }^{c}$ & $\overline{\mathrm{OR}}(95 \% \mathrm{Cl})^{d}$ & $\overline{p \text {-value }}{ }^{c}$ & & $\overline{\mathrm{OR}(95 \% \mathrm{Cl})^{\mathrm{b}}}$ & $\overline{p \text {-value }}{ }^{c}$ & $\overline{\text { OR }(95 \% \mathrm{Cl})^{\mathrm{e}} \quad p \text {-value }}$ \\
\hline & & $(0.699,1.067)$ & & $(0.740,1.173)$ & & & $(0.631,1.531)$ & & $(0.628,1.578)$ \\
\hline
\end{tabular}

$O R$ odds ratio, $C l$ confidence interval, $E R+$ Estrogen receptor positive, $P R+$ Progesterone receptor positive, ER- Estrogen receptor negative, $P R$ - Progesterone receptor negative

${ }^{a}$ Excludes 52 cases with unknown tumor hormone receptor status

${ }^{b}$ Models adjusted for age at baseline enrollment, race/ethnicity, region of residence

' For the categorical analysis, the $p$-values represent a test for linear trend with tertiles of PFAS modeled as a 3-level ordinal variable; for the continuous PFAS term, the $p$-value represents the $p$-value of the Wald-statistic for the $\beta$-coefficient for the PFAS modeled as a continuous term

${ }^{\mathrm{d}}$ ORs for ER+ or PR+ cases adjusted for: age at baseline enrollment, race/ethnicity, region of residence, date of blood draw, date of blood draw ${ }^{2}$, season of blood draw, total smoking pack-years, BMI, family history of breast cancer, age at first full-term pregnancy, menopausal status at blood draw and pork consumption e ORs for ER- and PR- cases adjusted for (except PFNA): age at baseline enrollment, race/ethnicity, region of residence, date of blood draw, date of blood draw ${ }^{2}$, season of blood draw and physical activity. ORs for PFNA adjusted for: age at baseline enrollment, race/ethnicity, region of residence, date of blood draw, date of blood draw ${ }^{2}$ and season of blood draw

f PFNA: ER+ or PR+ group excludes 26 cases with non-reportable serum values; ER- and PR- group excludes 4 with non-reportable serum values

may not be a valid representation of chronic PFAS exposures and the null results reported for most of the PFAS examined by the Danish study should not be regarded as evidence that chronic exposures are not related to breast cancer risk. On the other hand, pregnancy is considered an important window of susceptibility during which breast tissue may be particularly vulnerable to the deleterious effects of endocrine disrupting and carcinogenic compounds [73]. Findings from the Danish study suggest that other than possibly for PFOSA, PFAS exposures during this time do not impact breast cancer risk.

Overall we interpret the results from our study as null. Our analyses, however, indicated some suggestion of an inverse association between breast cancer risk and some PFASs, specifically PFUnDA and PFHxS, that appeared to be driven by risks among cases with non-hormonally responsive tumors. We find these results to be particularly perplexing. Given the body of literature on the potential estrogen disrupting properties of these compounds (with some studies suggesting anti-estrogenic properties), the most logical explanation for these findings would be that they are a reflection of anti-estrogenic activity. If this were the case, we would expect these effects to be more pronounced among cases with hormonally responsive tumors, as the etiology of these tumors is thought to be more strongly driven by hormonal factors than tumors that are not hormonally responsive $[74,75]$. We, in fact, found just the opposite, with more robust and pronounced inverse associations among the cases with hormonally non-responsive tumors and no statistically significant effects among the cases with $\mathrm{ER}+/ \mathrm{PR}+\mathrm{tu}-$ mors. Given the small number of cases upon which these estimates are based and the lack of a plausible biologic mechanism (at least based on our current state of knowledge), we regard these findings as either due to chance, or to be an artifact of our study design. In particular, the measurement of PFAS after treatment, coupled with our inability to account for treatment may be relevant.
To our knowledge, it is not known whether breast cancer treatment affects serum PFAS levels but it is conceivable that certain treatments, such as chemotherapy and hormonal therapy, could alter PFAS levels. One small study that compared serum levels of chlorinated hydrocarbons in breast cancer patients pre- and post-treatment reported significant differences in levels among patients who had received chemotherapy [76]. It is difficult to ascertain whether such findings are relevant to PFASs, which in contrast to the chlorinated hydrocarbons, are not lipophilic and would likely not be influenced by changes in body weight that could be associated with certain breast cancer treatments. The recommended course of treatment does differ for women with hormonally responsive and non-responsive tumors. Thus, it is plausible that the suggestive protective effects we observed for PFUnDA and PFHxS that appear to be confined to cases with ER-/PR- tumors could be a spurious artifact of the recommended treatment regimens more commonly prescribed for these breast cancer patients.

However, these suggestively inverse findings should not be entirely dismissed. A significant inverse association between breast cancer risk and PFHxS was also reported by the Danish study ( $\mathrm{RR}=0.66,95 \% \mathrm{CI}=0.47-0.94$ for logged serum concentrations) [51] and for PFOA in one of the C8 Science Panel studies (HR $=0.93,95 \% \mathrm{CI}=0.88-0.99$ for a logged estimated 10-year lagged cumulative PFOA serum concentration) [49]. Furthermore, these findings are consistent with evidence, although limited, from epidemiologic and animal studies that suggest PFAS exposures may delay the onset of puberty $[30,32,67]$ and lead to earlier menopause [77], both of which are conditions associated with reduced risks of breast cancer. In our study, neither age at menarche nor age at menopause were identified as significant confounders in our risk models and risks did not substantially differ when we stratified our analyses by categories of age at menarche or menopause (data not shown). 
Moreover, age at menarche did not correlate with serum PFAS levels (data not shown). A significant but very weak inverse correlation $(r<0.10)$, however, was seen between age at menopause and serum level of PFHxS but not PFUnDA (data not shown). Because the serum PFAS levels in our study were measured after menarche and menopause (in post-menopausal women), it is not possible to directly assess whether the suggestively protective effects we observed for some of the PFASs could be mediated by effects on the timing of menarcheal and menopausal onset.

When interpreting the results of our study it is important to consider potential mechanistic pathways. Three pathways have been proposed by which environmental chemicals such as the PFASs can impact breast cancer risk: 1) developmental toxicity mediated by endocrine disruption, leading to permanent morphologic changes in breast tissue and/or alterations in sex-steroid hormonal signaling pathways; 2) genotoxicity, either direct or indirect; and 3.) hormonal tumor promotion. Within each of these pathways, dose and timing of exposure are critical factors in determining effects [78].

For the PFASs, evidence is most compelling from both laboratory and human studies for developmental toxicity associated with exposures encountered during fetal through pre/peri-pubertal development [4, 30, 32, 67]. Our study, however, may not be particularly well-suited to evaluate breast cancer risks mediated through this pathway, at least for some of the PFASs, for which human exposures may not have become widespread until after our study subjects had passed through these important developmental windows of susceptibility. The first PFASs were introduced in the early 1950s. All the women in our study were born before 1972 and most (approximately 75\%) were born 1950 or earlier. Thus, the age structure of our study population makes it unlikely that many of our study participants would have been exposed to PFAS during fetal development. A lack of population-based historical biomonitoring data prior to the 1990s makes it difficult to ascertain how much opportunity for exposure our study participants would have had during the pre/peri-pubertal stages of development. While widespread human exposures first became widely recognized in the 1990s (thanks to the emergence of population-based biomonitoring programs), there is not sufficient historical biomonitoring data to clearly establish when human exposures became prevalent in the U.S. A recent temporal analysis of serum PFAS concentrations in three groups of California women collected in the 1960s, 1980s and 2009, however, suggests that elevated population exposures for at least some PFASs (specifically PFOS and PFHxS) may have been widespread as far back as the 1960s while widespread elevated exposures to other PFASs, particularly the longer chain perfluorocarboxylic acids such as PFOA and PFNA, may not have emerged until the 1980s or later [59].
Genotoxic effects are initiated through genetic damage which may lead to mutations that eventually progress to cancer. Mammary tissue appears to be most susceptible to the genotoxic effects of carcinogens during puberty and pregnancy when the mammary cells are not fully differentiated and are undergoing rapid proliferation [73]. However, exposures encountered at any time have the potential for carcinogenic genotoxicity. Although still a topic of some controversy, the current consensus based on evidence from laboratory data is that PFASs are not directly genotoxic but may be indirectly genotoxic through the formation of reactive oxygen species in response to oxidative stress [36, 39, 69, 78].

The mechanistic pathway for which our study is perhaps best suited to provide insight is that of hormonally-mediated tumor promotion. Many of the primary risk factors for breast cancer are thought to be mediated by cumulative lifetime exposure to high levels of endogenous estrogens [79] and the recent recognition of increased breast cancer risks associated with the use of menopausal hormone therapy [80] further highlights the potential impact of exposures to exogenous estrogens encountered later in life on breast cancer risk.

The estrogen disrupting properties of PFASs remain unclear. A fairly large body of laboratory studies, and to a lesser extent human studies, suggest myriad and complex potential estrogen disrupting effects which are far from well-understood. In laboratory studies, PFASs have been shown to both increase and decrease estradiol levels [4, 66, 67], with effects varying by species (and strains within species) $[4,32]$, by PFAS concentrations (with a dose-response relationship that is not necessarily monotonic) [66], and by specific PFAS [4, 51, 66]. Moreover, epidemiologic and in vitro data further suggest that the estrogen disrupting properties of PFASs may be highly dependent on co-exposure levels of endogenous estradiol. For example, PFOA exposures have been shown to be estrogenic in isolation but anti-estrogenic with co-exposure to high levels of estradiol $[66,67,81]$. Furthermore, recent epidemiologic data suggest that some PFASs may only act as estrogen disruptors among nulliparous women [72, 82]. For example, in a study of naturally cycling premenopausal women, Barrett and colleagues reported reductions in circulating estradiol associated with increases in serum PFOS concentrations but only among nulliparous, not parous women [72].

While we conducted a number of analyses among subsets of our study population designed to crudely capture groups of women who might have distinctly higher or lower levels of circulating endogenous estrogens (e.g. stratification by menopausal status, parity, categories of BMI, never/ever users of menopausal hormone therapy), these analyses proved to be non-illustrative. Without the ability to control for endogenous estrogen levels, both our generally null findings, and suggestively protective 
findings for some PFASs, may be an artifact of an inability to account for endogenous estrogen levels which could impact the estrogenic or anti-estrogenic effects of PFAS, if they exist.

The suggestion of a potentially protective effect associated with a few of the PFASs however warrants further attention, especially in light of toxicological evidence suggesting potential anti-estrogenic properties for some of these compounds under certain conditions. In particular, it appears that endogenous estrogen levels may be especially important in determining the mode of action of PFASs, including whether they exert estrogenic or anti-estrogenic properties. Future studies of this issue that can incorporate information about endogenous estrogen levels or genetic determinants of estrogen biosynthesis and metabolism would be particularly informative.

In summary, it is important to consider our findings in the context of the strengths and limitations of our study. The age structure of our study population limited our ability to evaluate risks associated with in utero exposures, which may be the most etiologically relevant time period for breast cancer risk. Furthermore, the collection of blood samples post-diagnosis and post-treatment calls into question the possibility of reverse causality. As with any epidemiologic case control study, the potential for selection bias and uncontrolled confounding also must be considered. However, our reliance on data collected from a case control study nested within an on-going cohort study that was specifically designed to study breast cancer, somewhat minimizes these concerns. The eligibility criteria for our study population was well-specified, cases and controls were carefully matched, and we saw little evidence of survival bias. The wealth of data collected on known breast cancer risk factors allowed for the evaluation of a comprehensive set of potential confounders.

\section{Conclusions}

The results from this study do not support an association between serum levels of PFASs and risk of breast cancer. Interpretation of these results, however, should not be overextended to conclude that PFASs are not of concern for breast cancer. In the context of the large body of laboratory data demonstrating endocrine disrupting properties of these PFASs at concentrations similar to those seen in some human populations, further study is warranted. It is especially important to elucidate mechanisms of action. Exposure to many of the PFASs examined in our study are declining due to regulatory and voluntary phase-outs enacted in response to heath concerns. These compounds however are being replaced with other PFASs that have not yet been studied [2, 23, 83]. A greater mechanistic understanding of how, and if, the PFASs influence breast cancer and other health outcomes is critical to avoiding "regrettable substitutions" of these compounds with replacement chemicals that may have similar toxicological properties and health consequences.

\section{Additional file}

Additional file 1: Table S1. Comparison of serum PFAS levels $(\mathrm{ng} / \mathrm{mL})$ in this study with levels in other epidemiologic studies of breast cancer risk. (DOCX $25 \mathrm{~kb})$

\section{Abbreviations}

BMI: Body mass index; CCR: California Cancer Registry; Cl: Confidence interval: CTS: California Teachers Study; ER-: Estrogen receptor negative; ER+: Estrogen receptor positive; EtFOSSA: 2-(N-Ethyl-perfluorooctane sulfonamido) acetic acid; HT: Hormone therapy; MeFOSAA: 2-(N-Methyl-perfluorooctane sulfonamido) acetic acid; OR: Odds ratio; PFAS: Per- and poly- fluoroalkyl substances; PFBS: Perfluorobutane sulfonic acid; PFDA: Perfluorodecanoic acid; PFDoDA: Perfluorododeconic acid; PFHpA: Perfluoroheptanoic acid; PFHxS: Perfluorohexane sulfonic acid; PFNA: Perfluorononanoic acid; PFOA: Perfluorooctanoic acid; PFOS: Perfluorooctane sulfonic acid; PFOSA: Perfluorooctane sulfonamide; PFUnDA: Perfluoroundecanoic acid; PR: Progesterone receptor negative; PR+: Progesterone receptor positive; US EPA: United States Environmental Protection Agency

\section{Acknowledgements}

We express our appreciation to all the participants in the California Teachers Study and to the phlebotomists, the researchers, analysts and staff who have contributed to the success of this research, including Weihong Guo, Suhash Harwani, Erika Houtz, Andrew Hertz, Minhthu Le, Christine Duffy, Jane Sullivan-Halley, Megan Johnson and the California Teachers Study Steering Committee members who continue to work on other aspects of the CTS cohort, including Jessica Clague deHart, Dennis Deapen, James V. Lacey Jr., Eunjung Lee, Huiyan Ma, Susan L. Neuhausen, Hannah Park, Richard Pinder, Sophia S. Wang, and Argyrios Ziogas.

\section{Funding}

This research was supported by funds provided by The Regents of the University of California, California Breast Cancer Research Program, Grant Number 16ZB-8501 and National Cancer Institute (NCl) of the National Institutes of Health $(\mathrm{NIH})$, Grant R01 CA77398. The opinions, findings, and conclusions herein are solely the responsibility of the authors and do not necessarily represent the official views of the $\mathrm{NIH}$, the California Department of Toxic Substances Control, the California Department of Public Health, the Regents of the University of California, or any of its programs.

Availability of data and materials

The datasets generated and/or analyzed during the current study are not publicly available due to confidentiality constraints as specified by the study's informed consent forms.

\section{Authors' contributions}

SH participated in the study design, helped plan and conceive the data analysis approach and drafted the manuscript; DG conducted the data analysis and reviewed the manuscript drafts; MW performed the PFAS assays and reviewed the manuscript drafts; JSP helped direct the PFAS assays and reviewed the manuscript drafts; MP planned and directed the PFAS assays and reviewed the manuscript drafts; HAC was responsible for conceiving and overseeing the CTS data and specimen collection activities and reviewed the manuscript drafts; LB was responsible for conceiving and overseeing the CTS data and specimen collection activities and reviewed the manuscript drafts; DON provided expertise to help direct the statistical analyses; PR was responsible for conceiving and overseeing the CTS data and specimen collection activities, conceived the overall study design, participated in directing data analysis, and reviewed manuscript drafts. All authors read and approved the final manuscript.

\section{Ethics approval and consent to participate}

Upon entry into the study, all participants provided their informed consent to use the data and biospecimens collected for research purposes such as this one. The use of human subjects was reviewed and approved by the California Health and Human Services Agency, Committee for the Protection of Human Subjects and the Institutional Review Boards of participating study institutions. 


\section{Consent for publication}

As part of their informed consent, participants agreed to the publication of research findings based on the data and biospecimens they provided.

\section{Competing interests}

The authors declare that they have no competing interests.

\section{Publisher's Note}

Springer Nature remains neutral with regard to jurisdictional claims in published maps and institutional affiliations.

\section{Author details}

${ }^{1}$ Cancer Prevention Institute of California, Berkeley, CA, USA. ${ }^{2}$ Department of Epidemiology and Biostatistics, University of California San Francisco, 2001 Center Street, Suite 700, Berkeley 94704, California, USA. ${ }^{3}$ Environmental Chemistry Laboratory, Department of Toxic Substances Control, Berkeley, CA, USA. ${ }^{4}$ Department of Population Sciences, Beckman Research Institute of the City of Hope, Duarte, CA, USA. ${ }^{5}$ Department of Epidemiology, School of Medicine, University of California Irvine, Irvine, CA, USA. ${ }^{6}$ Department of Health Research and Policy, Stanford University School of Medicine, Stanford, CA, USA.

\section{Received: 10 May 2018 Accepted: 13 November 2018}

Published online: 27 November 2018

\section{References}

1. Agency for Toxic Substances and Disease Registry Draft for Public Comment June 2018. Centers for Disease Control and Prevention, Atlanta. Available from: http://www.atsdr.cdc.gov/toxprofiles/tp200.pdf. Cited 20 Nov 2017.

2. Land M, de Witt CA, Cousins IT, Herzke D, Johansson J, Martin JW. What is the effect of phasing out long-chain per-and polyfluoroalkyl substances on the concentrations of perfluoroalkyl acids and their precursors in the environment? A systematic review protocol. Environ Evid. 2015;4(4):1-13.

3. Lindstrom AB, Strynar MJ, Libelo EL. Polyfluorinated compounds: past, present, and future. Environ Sci Technol. 2011;45(19):7954-61.

4. Lau C. Perfluorinated compounds. In: Luch A, editor. Molecular, clinical and environmental toxicology. Basel: Springer; 2012. p. 47-86.

5. Lau C, Anitole K, Hodes C, Lai D, Pfahles-Hutchens A, Seed J. Perfluoroalkyl acids: a review of monitoring and toxicological findings. Toxicol Sci. 2007; 99(2):366-94.

6. Wang Z, Cousins IT, Scheringer M, Buck RC, Hungerbuhler K. Global emission inventories for C4-C14 perfluoroalkyl carboxylic acid (PFCA) homologues from 1951 to 2030, Part I: production and emissions from quantifiable sources. Environ Int. 2014;70:62-75.

7. Wang Z, Cousins IT, Scheringer M, Buck RC, Hungerbuhler K. Global emission inventories for (4-C14 perfluoroalkyl carboxylic acid (PFCA) homologues from 1951 to 2030, part II: the remaining pieces of the puzzle. Environ Int. 2014;69:166-76.

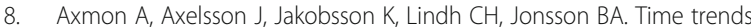
between 1987 and 2007 for perfluoroalkyl acids in plasma from Swedish women. Chemosphere. 2014;102:61-7.

9. Bjerregaard-Olesen C, Bach CC, Long M, et al. Time trends of perfluorinated alkyl acids in serum from Danish pregnant women 2008-2013. Environ Int. 2016;91:14-21

10. Calafat AM, Kuklenyik Z, Reidy JA, Caudill SP, Tully JS, Needham LL. Serum concentrations of 11 polyfluoroalkyl compounds in the u.s. population: data from the national health and nutrition examination survey (NHANES). Environ Sci Technol. 2007:41(7):2237-42

11. Calafat AM, Wong LY, Kuklenyik Z, Reidy JA, Needham LL. Polyfluoroalkyl chemicals in the U.S. population: data from the National Health and nutrition examination survey (NHANES) 2003-2004 and comparisons with NHANES 1999-2000. Environ Health Perspect. 2007;115(11):1596-602

12. Biomonitoring California. California Department of Public Health, Department of Toxic Substances Control, Office of Health Hazard Assessment. CA2012-2016; Available from: http://www.biomonitoring.ca. gov/. Cited 15 Nov 2017

13. Centers for Disease Control and Prevention National Center for Health Statistics. NHANES 2011-2012 Laboratory Data. 2014; Available from: https:// wwwn.cdc.gov/nchs/nhanes/search/datapage.aspx?Component= Laboratory\&CycleBeginYear=2011. Cited 14 June 2017.
14. Centers for Disease Control and Prevention National Center for Health Statistics. NHANES 2013-2014 Laboratory Data. 2017; Available from: https:// wwwn.cdc.gov/nchs/nhanes/search/datapage.aspx?Component= Laboratory\&CycleBeginYear=2013. Cited 2017 July 2017.

15. Cho CR, Lam NH, Cho BM, Kannan K, Cho HS. Concentration and correlations of perfluoroalkyl substances in whole blood among subjects from three different geographical areas in Korea. Sci Total Environ. 2015; 512-513:397-405

16. Dobraca D, Israel L, McNeel S, et al. Biomonitoring in California firefighters: metals and perfluorinated chemicals. J Occup Environ Med. 2015;57(1):8897.

17. Eriksson U, Mueller JF, Toms LL, Hobson P, Karrman A. Temporal trends of PFSAs, PFCAs and selected precursors in Australian serum from 2002 to 2013. Environ Pollut. 2017;220(Pt A):168-77.

18. Fromme H, Tittlemier SA, Volkel W, Wilhelm M, Twardella D. Perfluorinated compounds--exposure assessment for the general population in Western countries. Int J Hyg Environ Health. 2009;212(3):239-70.

19. Kannan K, Corsolini S, Falandysz J, et al. Perfluorooctanesulfonate and related fluorochemicals in human blood from several countries. Environ Sci Technol. 2004;38(17):4489-95.

20. Kato K, Wong LY, Jia LT, Kuklenyik Z, Calafat AM. Trends in exposure to polyfluoroalkyl chemicals in the U.S. population: 1999-2008. Environ Sci Technol. 2011;45(19):8037-45.

21. Miralles-Marco A, Harrad S. Perfluorooctane sulfonate: a review of human exposure, biomonitoring and the environmental forensics utility of its chirality and isomer distribution. Environ Int. 2015;77:148-59.

22. Zhang Y, Beesoon S, Zhu L, Martin JW. Biomonitoring of perfluoroalkyl acids in human urine and estimates of biological half-life. Environ Sci Technol. 2013:47(18):10619-27.

23. Wang Z, DeWitt JC, Higgins CP, Cousins IT. A never-ending story of per- and Polyfluoroalkyl substances (PFASs)? Environ Sci Technol. 2017;51(5):2508-18.

24. Zhang L, Liu J, Hu J, et al. The inventory of sources, environmental releases and risk assessment for perfluorooctane sulfonate in China. Environ Pollut. 2012;165:193-8.

25. C8 Science Panel. Probable Links Reports. 2012 [February 2016]; Available from: http://www.c8sciencepanel.org/prob link.html. Cited 20 Nov 2018.

26. DeWitt JC, Peden-Adams MM, Keller JM, Germolec DR. Immunotoxicity of perfluorinated compounds: recent developments. Toxicol Pathol. 2012;40(2): 300-11.

27. Domingo $\mathrm{J}$. Health risks of dietary exposure to perfluorinated compounds. Environ Int. 2012:40:187-95.

28. Ganesan S, Vasudevan N. Impacts of perfluorinated compounds on human health. Bull Environ Pharmacol Life Sci. 2015:4(7):183-91.

29. Grandjean P, Clapp R. Perfluorinated alkyl substances: emerging insights into health risks. New Solut. 2015:25(2):147-63.

30. Reed CE, Fenton SE. Effects of PFOA on endocrine-related systems. In: DeWitt J, editor. Toxicological effects of Perfluoroalkyl and Polyfluoroalkyl substances molecular and integrative toxicology. Cham: Humana Press; 2015. p. 249-64

31. Steenland K, Fletcher T, Savitz DA. Epidemiologic evidence on the health effects of perfluorooctanoic acid (PFOA). Environ Health Perspect. 2010; 118(8):1100-8

32. White SS, Fenton SE, Hines EP. Endocrine disrupting properties of perfluorooctanoic acid. J Steroid Biochem Mol Biol. 2011;127(1-2):16-26.

33. Institute of Medicine of the National Academies. Breast Cancer and the Environment: a life course approach. Washington, D.C.: National Academy of Science; 2014.

34. Rudel RA, Ackerman JM, Attfield KR, Brody JG. New exposure biomarkers as tools for breast cancer epidemiology, biomonitoring, and prevention: a systematic approach based on animal evidence. Environ Health Perspect. 2014;122(9):881-95.

35. Schwarzman MR, Ackerman JM, Dairkee SH, et al. Screening for chemical contributions to breast Cancer risk: a case study for chemical safety evaluation. Environ Health Perspect. 2015:123(12):1255-64.

36. Arrieta-Cortes R, Farias P, Hoyo-Vadillo C, Kleiche-Dray M. Carcinogenic risk of emerging persistent organic pollutant perfluorooctane sulfonate (PFOS): a proposal of classification. Regul Toxicol Pharmacol. 2017;83:66-80.

37. Chang ET, Adami HO, Boffetta P, Wedner HJ, Mandel JS. A critical review of perfluorooctanoate and perfluorooctanesulfonate exposure and immunological health conditions in humans. Crit Rev Toxicol. 2016:46(4): 279-331. 
38. Benbrahim-Tallaa L, Lauby-Secretan B, Loomis D, et al. Carcinogenicity of perfluorooctanoic acid, tetrafluoroethylene, dichloromethane, 1,2dichloropropane, and 1,3-propane sultone. Lancet Oncol. 2014;15(9):924-5.

39. World Health Organization (WHO) International Agency for Research on Cancer (IARC). IARC Monographs on the Evaluation of Carcinogenic Risks to Humans. Some Chemicals Used as Solvents and in Polymer Manufacture. Volume 110 (2017). Lyon, France; 2017 [updated December 22, 2016; cited 2018]; Available from: http://monographs.iarc.fr/ENG/Monographs/vol110/ index.php. Cited 20 November 2018.

40. United States Environmental Protection Agency (US EPA). Draft Risk Assessment of Potential Human Health Effects ASsocated with PFOA and Its Salts. Washington, D.C.: US EPA SAB; 2006.

41. Alexander $\mathrm{BH}$, Olsen GW. Bladder cancer in perfluorooctanesulfonyl fluoride manufacturing workers. Ann Epidemiol. 2007;17(6):471-8.

42. Alexander BH, Olsen GW, Burris JM, Mandel JH, Mandel JS. Mortality of employees of a perfluorooctanesulphonyl fluoride manufacturing facility. Occup Environ Med. 2003;60(10):722-9.

43. Consonni D, Straif K, Symons JM, et al. Cancer risk among tetrafluoroethylene synthesis and polymerization workers. Am J Epidemiol. 2013;178(3):350-8.

44. Gilliland FD, Mandel JS. Mortality among employees of a perfluorooctanoic acid production plant. J Occup Med. 1993;35(9):950-4.

45. Leonard RC, Kreckmann KH, Sakr CJ, Symons JM. Retrospective cohort mortality study of workers in a polymer production plant including a reference population of regional workers. Ann Epidemiol. 2008;18(1):15-22.

46. Lundin Jl, Alexander BH, Olsen GW, Church TR. Ammonium perfluorooctanoate production and occupational mortality. Epidemiology. 2009;20(6):921-8.

47. Raleigh KK, Alexander BH, Olsen GW, et al. Mortality and cancer incidence in ammonium perfluorooctanoate production workers. Occup Environ Med. 2014;71(7):500-6.

48. Steenland K, Woskie S. Cohort mortality study of workers exposed to perfluorooctanoic acid. Am J Epidemiol. 2012;176(10):909-17.

49. Barry V, Winquist A, Steenland K. Perfluorooctanoic acid (PFOA) exposures and incident cancers among adults living near a chemical plant. Environ Health Perspect. 2013;121(11-12):1313-8.

50. Vieira VM, Hoffman K, Shin HM, Weinberg JM, Webster TF, Fletcher T. Perfluorooctanoic acid exposure and cancer outcomes in a contaminated community: a geographic analysis. Environ Health Perspect. 2013;121(3): 318-23.

51. Bonefeld-Jorgensen EC, Long M, Fredslund SO, Bossi R, Olsen J. Breast cancer risk after exposure to perfluorinated compounds in Danish women: a case-control study nested in the Danish National Birth Cohort. Cancer Causes Control. 2014;25(11):1439-48.

52. Ghisari M, Long M, Roge DM, Olsen J, Bonefeld-Jorgensen EC. Polymorphism in xenobiotic and estrogen metabolizing genes, exposure to perfluorinated compounds and subsequent breast cancer risk: a nested case-control study in the Danish National Birth Cohort. Environ Res. 2017; 154:325-33.

53. Bonefeld-Jorgensen EC, Long M, Bossi R, et al. Perfluorinated compounds are related to breast cancer risk in Greenlandic Inuit: a case control study. Environ Health. 2011;10:88.

54. Ghisari M, Eiberg H, Long M, Bonefeld-Jorgensen EC. Polymorphisms in phase I and phase II genes and breast cancer risk and relations to persistent organic pollutant exposure: a case-control study in Inuit women. Environ Health. 2014;13(1):19.

55. Wielsoe M, Kern P, Bonefeld-Jorgensen EC. Serum levels of environmental pollutants is a risk factor for breast cancer in Inuit: a case control study. Environ Health. 2017;16(1):56

56. Bernstein L, Allen M, Anton-Culver $H$, et al. High breast cancer incidence rates among California teachers: results from the California teachers study (United States). Cancer Causes Control. 2002;13(7):625-35.

57. California Cancer Registry (CCR) California Department of Health. California Cancer Registry FAQ. 2017; Available from: http://www.ccrcal.org/Inside_ CCR/FAQ.shtml. Cited 1 Feb 2018.

58. Hurley S, Goldberg D, Wang M, et al. Time trends in per- and Polyfluoroalkyl substances (PFASs) in California women: declining serum levels, 2011-2015. Environ Sci Technol. 2018;52(1):277-87.

59. Wang M, Park JS, Petreas M. Temporal changes in the levels of perfluorinated compounds in California women's serum over the past 50 years. Environ Sci Technol. 2011;45(17):7510-6.
60. Block G, Hartman AM, Dresser CM, Carroll MD, Gannon J, Gardner L. A databased approach to diet questionnaire design and testing. Am J Epidemiol. 1986;124(3):453-69.

61. Horn-Ross PL, Hoggatt KJ, West DW, et al. Recent diet and breast cancer risk: the California teachers study (USA). Cancer Causes Control. 2002;13(5): 407-15.

62. Reynolds P, Hurley S, Goldberg DE, et al. Regional variations in breast cancer among California teachers. Epidemiology. 2004;15(6):746-54.

63. Reynolds P, Hurley SE, Quach AT, et al. Regional variations in breast cancer incidence among California women, 1988-1997. Cancer Causes Control. 2005;16(2):139-50.

64. Centers for Disease Control and Prevention. Fourth National Report on Human Exposures to Environmental Chemicals Updated Tables, January 2017, vol. 1. Atlanta: Department of Health and Human Services; 2009.

65. Hornung RW, Reed LW. Estimation of average concentration in the presence of nondetectable values. Appl Occup Environ Hyg. 1990;5(1):46-51.

66. Henry ND, Fair PA. Comparison of in vitro cytotoxicity, estrogenicity and anti-estrogenicity of triclosan, perfluorooctane sulfonate and perfluorooctanoic acid. J Appl Toxicol. 2013;33(4):265-72.

67. Macon MB, Fenton SE. Endocrine disruptors and the breast: early life effects and later life disease. J Mammary Gland Biol Neoplasia. 2013;18(1):43-61.

68. Kato K, Ye X, Calafat AM. Chapter 3: PFASs in general population. In: DeWitt JC, editor. Toxicological effects of Perfluoroalkyl and Polyfluoroalkyl substances, molecular and integrative toxicology. Switzerland: Spring International Publishing; 2015.

69. Bonefeld-Jorgensen EC, Ghisari M, Wielsoe M, Bjerregaard-Olesen C, Kjeldsen LS, Long M. Biomonitoring and hormone-disrupting effect biomarkers of persistent organic pollutants in vitro and ex vivo. Basic Clin Pharmacol Toxicol. 2014;115(1):118-28.

70. Dai Y, Liu J, Yuan E, et al. Gestational age-specific reference intervals for 15 biochemical measurands during normal pregnancy in China. Ann Clin Biochem. 2017. https://doi.org/10.1177/0004563217738801.

71. Woodruff TJ, Zota AR, Schwartz JM. Environmental chemicals in pregnant women in the United States: NHANES 2003-2004. Environ Health Perspect. 2011;119(6):878-85.

72. Barrett ES, Chen C, Thurston SW, et al. Perfluoroalkyl substances and ovarian hormone concentrations in naturally cycling women. Fertil Steril. 2015; 103(5):1261-70 e3.

73. Russo J, Russo IH. Development of the human breast. Maturitas. 2004;49(1):2-15.

74. Althuis MD, Fergenbaum JH, Garcia-Closas M, Brinton LA, Madigan MP, Sherman ME. Etiology of hormone receptor-defined breast cancer: a systematic review of the literature. Cancer Epidemiol Biomark Prev. 2004; 13(10):1558-68.

75. Ma H, Bernstein L, Pike MC, Ursin G. Reproductive factors and breast cancer risk according to joint estrogen and progesterone receptor status: a metaanalysis of epidemiological studies. Breast Cancer Res. 2006;8(4):R43.

76. Gammon MD, Wolff MS, Neugut Al, et al. Treatment for breast cancer and blood levels of chlorinated hydrocarbons. Cancer Epidemiol Biomark Prev. 1996;5(6):467-71.

77. Taylor KW, Hoffman K, Thayer KA, Daniels JL. Polyfluoroalkyl chemicals and menopause among women 20-65 years of age (NHANES). Environ Health Perspect. 2014;122(2):145-50.

78. Rodgers KM, Udesky JO, Rudel RA, Brody JG. Environmental chemicals and breast cancer: an updated review of epidemiological literature informed by biological mechanisms. Environ Res. 2018;160:152-82.

79. Yager JD, Davidson NE. Estrogen carcinogenesis in breast cancer. N Engl J Med. 2006;354(3):270-82.

80. Davey DA. Update: estrogen and estrogen plus progestin therapy in the care of women at and after the menopause. Womens Health (Lond). 2012; $8(2): 169-89$

81. Liu C, Du Y, Zhou B. Evaluation of estrogenic activities and mechanism of action of perfluorinated chemicals determined by vitellogenin induction in primary cultured tilapia hepatocytes. Aquat Toxicol. 2007;85(4):267-77.

82. Knox SS, Jackson T, Javins B, Frisbee SJ, Shankar A, Ducatman AM. Implications of early menopause in women exposed to perfluorocarbons. J Clin Endocrinol Metab. 2011;96(6):1747-53.

83. Cousins IT, Vestergren $R$, Wang Z, Scheringer M, McLachlan MS. The precautionary principle and chemicals management: the example of perfluoroalkyl acids in groundwater. Environ Int. 2016;94:331-40. 